\title{
Possible instabilities in quadratic and cubic nodal line fermion systems with correlated interactions
}

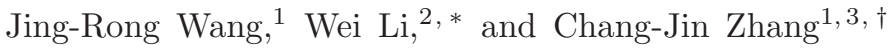 \\ ${ }^{1}$ Anhui Province Key Laboratory of Condensed Matter Physics at Extreme Conditions, \\ High Magnetic Field Laboratory of Anhui Province, \\ Chinese Academy of Sciences, Hefei 230031, China \\ ${ }^{2}$ Key Laboratory of Materials Physics, Institute of Solid State Physics, \\ Chinese Academy of Sciences, Hefei 230031, China \\ ${ }^{3}$ Institute of Physical Science and Information Technology, Anhui University, Hefei 230601, China
}

\begin{abstract}
Influence of short-range four-fermion interactions on quadratic and cubic nodal line fermion systems is studied by renormalization group theory. It is found that arbitrarily weak four-fermion interaction could drive quadratic or cubic nodal line fermion system to a new phase. According to the initial conditions and value of fermion flavor, the system may appear three kinds of instabilities. First, quadratic or cubic nodal line is split into conventional nodal lines, thus the system becomes nodal line semimetal. Second, finite excitonic gap is generated, and the system becomes an excitonic insulator. Third, the system is driven into superconducting phase. Thus, quadratic and cubic nodal line fermion systems are rare strong correlated fermion systems in three dimension under the influence of four-fermion interactions. These theoretical results may be verified in the candidates for quadratic and cubic nodal line fermion systems.
\end{abstract}

\section{INTRODUCTION}

Study about topological semimetals (SMs) is one of the most important fields of modern condensed matter physics [1 15]. On the one hand, some SMs have wide potential industrial applications due to their fantastic properties, such as large thermoelectric power [16, 17]. On the other hand, some SMs provide a platform to verify certain important concepts in high energy physics, due to that their low-energy fermion excitations resemble the elementary particles [1-15].

According to energy dispersion of the fermion excitations and topological property of the system, SMs can be classified as Dirac SM (DSM), Weyl SM (WSM), multiWSMs, semi-DSM, Luttinger SM, nodal line SM (NLSM) etc. [9]. Graphene is a prototypical two dimensional (2D) DSM. Angle-resolved photoemission spectroscopy (ARPES) experiments have confirmed that $\mathrm{Cd}_{3} \mathrm{As}_{2}$ and $\mathrm{Na}_{3} \mathrm{Bi}$ are 3D DSM 18, 19], and TaAs, TaP, NbAs, NbP are WSMs [8, 11, 20, 21]. NLSM has been realized in $\mathrm{PbTaSe}_{2}$, ZrSiS, ZrSiSe, HfSiS, and $\mathrm{TiB}_{2}$ according to ARPES measurements 22 28].

In SMs, the dimension of Fermi surface is at least two less than the dimension of the system. This characteristic is different from conventional metals, in which the dimension of Fermi surface is one less than the dimension of the system [29]. In DSM, WSM, multi-WSMs, semi-DSM, and Luttinger SM, the Fermi surface is composed by discrete points, where conduction and valence bands touch each other in the Brillouin zone. Whereas, the Fermi surafce of NLSM is a line in the 3D Brillouin zone. Due to the abovementioned characteristic of SMs,

\footnotetext{
*Corresponding author: wliustc@theory.issp.ac.cn

${ }^{\dagger}$ Corresponding author: zhangcj@hmfl.ac.cn
}

the density of states (DOS) of SMs vanishes at the Fermi level.

Influence of interactions on SMs is an important and nontrivial question, which attracts much attentions [1, 30 66]. Due to the vanishing DOS, short-range fourfermion interaction is irrelevant in SM if it is weak, but may drive a quantum phase transition (QPT) to a new phase if the interaction strength is large enough [57 66]. There have been studies on the effects of four-fermion interactions in SMs including 2D DSM [57, 58], 3D DSM [59], WSM 60, 61, multi-WSMs 61], semi-DSM [62, 63], Luttinger SM 64], and NLSM 65 67]. These studies showed that the SMs may be driven to different phases according to the types of four-fermion interactions. Additionally, the influence of four-fermion interactions is closely related to the fermion dispersion and topological property of the system.

In NLSM, the fermion dispersion is linear within the $x-y$ plane and also linear along the $z$ axis [47, 48, 6570]. For NLSM, DOS takes the form $\rho(\omega) \sim \omega$, which vanishes at the Fermi level, i.e., $\rho(0)=0$. Roy has analyzed the possible QPTs in NLSM under the influence of four-fermion interactions [66].

Recently, $\mathrm{Yu}$ et al. proposed that quadratic and cubic nodal line fermion (NLF) systems could be realized in some materials 71]. In these materials, the Fermi surface is also a line in the $3 \mathrm{D}$ Brillouin zone, which is same as NLSM. However, the fermion dispersion is quadratic (cubic) within the $x-y$ plane and also quadratic (cubic) along the $z$ axis [71, 72]. Accordingly, DOS satisfies $\rho(\omega) \sim \omega^{0}$ in quadratic NLF system, and $\rho(\omega) \sim \omega^{-1 / 3}$ in cubic NLF system. Thus, the influence of four-fermion interactions on quadratic and cubic NLF systems could be substantially different from the one in NLSM. This is an interesting and urgent question, which needs comprehensive study.

In this article, we resolve this question through renor- 
malization group (RG) theory 73 . We find that quadratic and cubic NLF fermion systems are unstable to short-range four-fermion interactions. We show that arbitrarily weak four-fermion interaction could drive quadratic or cubic NLF system to a new phase. According to the initial conditions and value of fermion flavor, the system may appear three kinds of instabilities. First, the quadratic or cubic nodal line is split into conventional nodal lines, and the system becomes a NLSM. Second, finite excitonic gap is generated, then the system becomes an excitonic insulator. Third, the system is driven into a superconducting phase.

The rest of paper is structured as follows. The model is presented in Sec. II. In Sec. III we analyze the physical meaning of various fermion bilinears, which may be generated by the four-fermion interactions. We perform the mean field calculation in Sec. IV In Sec. V] we show RG equations of the model parameters and numerical results of the RG equations. The behaviors of observable quantities in different phases are discussed in Sec. VI. In Sec. VII, we discuss the role of geometry of nodal line. The main results are summarized in Sec. VIII, The detailed calculation and derivation for the $\mathrm{RG}$ equations are given in the Appendices.

\section{MODEL}

In Ref. 71], after performing a symmetry analysis over all 230 space groups for solid systems with spin-orbit coupling and time-reversal symmetry, $\mathrm{Yu}$ et al. found that quadratic and cubic nodal lines can be stabilized by crystalline symmetries. For convenience, in Appendix A we show lattice models for quadratic and cubic NLF systems, and derive the low-energy effective models. In the following, our analysis will focus on the low-energy effective models directly.

The Hamiltonian density for free quadratic NLF system is given by

$$
\mathcal{H}_{0}^{q}(\mathbf{k})=A\left[\left(k_{r}^{2}-k_{z}^{2}\right) \sigma_{1}+2 k_{r} k_{z} \sigma_{2}\right],
$$

where $k_{r}=k_{\perp}-k_{F}$ with $k_{\perp}=\sqrt{k_{x}^{2}+k_{y}^{2}}$. $A$ is a model parameter. $\sigma_{1,2,3}$ are the standard Pauli matrices. The energy spectrum for quadratic NLF is

$$
E_{q}(\mathbf{k})= \pm A\left(k_{r}^{2}+k_{z}^{2}\right) \text {. }
$$

For simplicity, here we do not consider anisotropy of the fermion dispersion along $k_{r}$ and $k_{z}$. The qualitative conclusions will not be changed if this anisotropy is incorporated.

The Hamiltonian density for cubic NLF system can be written as

$$
\mathcal{H}_{0}^{c}(\mathbf{k})=B\left[\left(k_{r}^{3}-3 k_{r} k_{z}^{2}\right) \sigma_{1}+\left(k_{z}^{3}-3 k_{z} k_{r}^{2}\right) \sigma_{2}\right],
$$

with $B$ being a model parameter. The energy dispersion for cubic NLF takes the form

$$
E_{c}(\mathbf{k})= \pm B\left(k_{r}^{2}+k_{z}^{2}\right)^{3 / 2} .
$$

The Pauli matrices $\sigma_{i}$ act on the sublattice space of freedom. Both the Hamiltonian densities $\mathcal{H}_{0}^{q}$ and $\mathcal{H}_{0}^{c}$ satisfy the chiral symmetry $\left\{\mathcal{H}_{0}^{q, c}, \sigma_{3}\right\}=0$. Once a term $\mathcal{H}_{\Delta_{3}}=\Delta_{3} \sigma_{3}$ is generated, the fermions become gapped and the chiral symmetry is broken [71].

We consider the four-fermion interactions described by the action

$$
\begin{aligned}
S_{\psi^{4}}= & \frac{1}{N} \sum_{i=0}^{3} \lambda_{i} \int \frac{d \omega_{1}}{2 \pi} \frac{d^{3} \mathbf{k}_{1}}{(2 \pi)^{3}} \frac{d \omega_{2}}{2 \pi} \frac{d^{3} \mathbf{k}_{2}}{(2 \pi)^{3}} \frac{d \omega_{3}}{2 \pi} \frac{d^{3} \mathbf{k}_{3}}{(2 \pi)^{3}} \\
& \times \psi^{\dagger}\left(\omega_{1}, \mathbf{k}_{1}\right) \sigma_{i} \psi\left(\omega_{2}, \mathbf{k}_{2}\right) \psi^{\dagger}\left(\omega_{3}, \mathbf{k}_{3}\right) \sigma_{i} \\
& \times \psi\left(\omega_{1}-\omega_{2}+\omega_{3}, \mathbf{k}_{1}-\mathbf{k}_{2}+\mathbf{k}_{3}\right),
\end{aligned}
$$

where $\lambda_{i}$ with $i=0,1,2,3$ are the four-fermion coupling parameters, and $N$ is the fermion flavor. The fermion flavor $N$ represents the degeneracy of the real spin. In the calculation, we take $N$ as a general turning parameter. The physical value of $N$ is $N=2 . \sigma_{0}$ is the identity matrix. In the following, we are only interest in the case that the initial value $\lambda_{i, 0}$ satisfies $\lambda_{i, 0}>0$, namely the interaction is repulsive initially.

\section{PHYSICAL MEANING OF FERMION BILINEARS}

Decoupling the four-fermion interactions, we could get four different fermion bilinears $\psi^{\dagger} \sigma_{0} \psi, \psi^{\dagger} \sigma_{1} \psi, \psi^{\dagger} \sigma_{2} \psi$, and $\psi^{\dagger} \sigma_{3} \psi$. The expectation values of these bilinears are given by

$$
\begin{aligned}
& \Delta_{0}=\left\langle\psi^{\dagger} \sigma_{0} \psi\right\rangle, \\
& \Delta_{1}=\left\langle\psi^{\dagger} \sigma_{1} \psi\right\rangle, \\
& \Delta_{2}=\left\langle\psi^{\dagger} \sigma_{2} \psi\right\rangle, \\
& \Delta_{3}=\left\langle\psi^{\dagger} \sigma_{3} \psi\right\rangle .
\end{aligned}
$$

$\langle\ldots\rangle$ represents taking mean value on the ground state of total Hamiltonian. They have different physical meanings. If $\Delta_{0}$ becomes finite, the Fermi level is modified, and the Fermi surface is changed from 1D nodal line to $2 \mathrm{D}$ tube, since $\Delta_{0}$ represents the chemical potential.

The original nodal line is gapless for $\left(k_{r}, k_{z}\right)=(0,0)$. For quadratic NLF system, if $\Delta_{1}$ becomes finite, the original nodal line with quadratic dispersion is split into two conventional nodal lines with linear dispersion. These two conventional nodal lines are gapless for the cases

$$
\left(k_{a r}, k_{a z}\right)=\left(0,\left(\Delta_{1} / A\right)^{1 / 2}\right) \text {, }
$$

and

$$
\left(k_{b r}, k_{b z}\right)=\left(0,-\left(\Delta_{1} / A\right)^{1 / 2}\right) .
$$

Around these two nodal lines, the fermion dispersion can be written as

$$
E= \pm \sqrt{4 A \Delta_{1}\left(K_{r}^{2}+K_{z}^{2}\right)}
$$



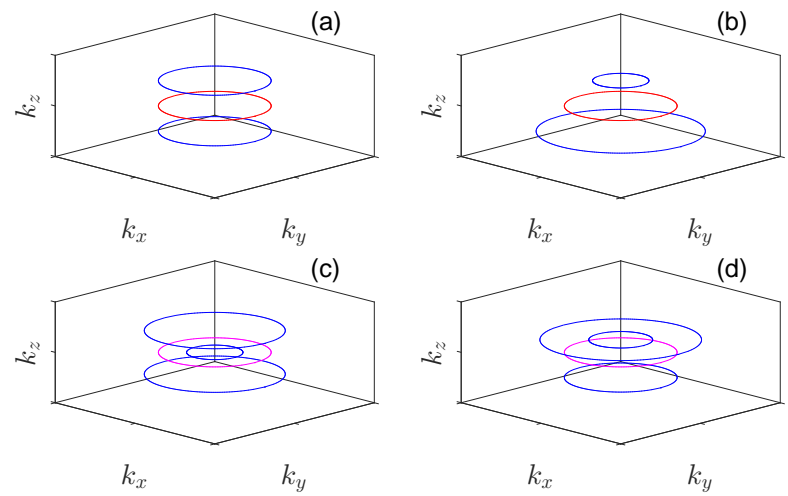

FIG. 1: (a) and (b) Splitting of quadratic nodal line into conventional nodal lines in the presence of $\Delta_{1}$ and $\Delta_{2}$ respectively. (c) and (d) Splitting of cubic nodal line into conventional nodal lines in the presence of $\Delta_{1}$ and $\Delta_{2}$ respectively. Red, magenta, and blue lines correspond to quadratic, cubic, and conventional nodal lines respectively.

where $K_{r}$ and $K_{z}$ are the momentum components relative to the nodal lines.

For cubic NLF system, if $\Delta_{1}$ becomes finite, one cubic nodal line is split into three conventional nodal lines, which are determined by

$$
\begin{aligned}
\left(k_{a r}, k_{a z}\right) & =\left(-\left(\Delta_{1} / B\right)^{1 / 3}, 0\right) \\
\left(k_{b r}, k_{b z}\right) & =\left(\frac{1}{2}\left(\Delta_{1} / B\right)^{1 / 3}, \frac{\sqrt{3}}{2}\left(\Delta_{1} / B\right)^{1 / 3}\right) \\
\left(k_{c r}, k_{c r}\right) & =\left(\frac{1}{2}\left(\Delta_{1} / B\right)^{1 / 3},-\frac{\sqrt{3}}{2}\left(\Delta_{1} / B\right)^{1 / 3}\right)
\end{aligned}
$$

The energy dispersion around these three nodal lines can be expressed as

$$
E= \pm \sqrt{9 B^{2 / 3} \Delta_{1}^{4 / 3}\left(K_{r}^{2}+K_{z}^{2}\right)} .
$$

For quadratic NLF system, if $\Delta_{2}$ acquires finite value, one quadratic nodal line is split into two conventional nodal lines, which satisfy

$$
\begin{aligned}
\left(k_{a r}, k_{a z}\right) & =\left(\left(\Delta_{2} /(2 A)\right)^{1 / 2},-\left(\Delta_{2} /(2 A)\right)^{1 / 2}\right), \\
\left(k_{b r}, k_{b z}\right) & =\left(-\left(\Delta_{2} /(2 A)\right)^{1 / 2},\left(\Delta_{2} /(2 A)\right)^{1 / 2}\right) .
\end{aligned}
$$

The energy dispersion of the fermions around these two nodal lines reads as

$$
E= \pm \sqrt{4 A \Delta_{2}\left(K_{r}^{2}+K_{z}^{2}\right)} .
$$

For cubic NLF system, if $\Delta_{2}$ becomes finite, one cubic nodal line is split into three conventional nodal lines. The corresponding conditions can be obtained through Eq. (13)-(15) by employing $k_{j z} \leftrightarrow k_{j r}$ with $j=a, b, c$ and $\Delta_{1} \rightarrow \Delta_{2}$. Around these three nodal lines, the fermion dispersion can be expressed by Eq. (16) through utilizing $\Delta_{1} \rightarrow \Delta_{2}$. .
If $\Delta_{3}$ acquires finite value, the energy dispersions for quadratic and cubic NLF systems can be written as

$$
E_{q, c}\left(\mathbf{k}, \Delta_{3}\right)= \pm \sqrt{E_{q, c}^{2}(\mathbf{k})+\Delta_{3}^{2}}
$$

where $E_{q}(\mathbf{k})$ and $E_{c}(\mathbf{k})$ are given by Eqs. (2) and (4) respectively. We can find that the fermion dispersion becomes gapped once $\Delta_{3}$ becomes finite. Physically, it suggests that the system is driven into excitonic insulating phase.

For convenience, we show the splitting of quadratic and cubic nodal lines to conventional nodal lines in the presence of $\Delta_{1}$ or $\Delta_{2}$ by the schematic diagrams in Fig. 1.

For a fermion system under the influence of fourfermion interaction $\lambda\left(\psi^{\dagger} \Gamma \psi\right)^{2}$ where $\Gamma$ is a matrix, if the RG analysis shows that the four-fermion coupling strength $\lambda$ approaches to infinity at a finite running parameter $\ell_{c}$, it indicates that the system becomes unstable under the energy scale

$$
\Lambda_{U S}=\Lambda e^{-\ell_{c}}
$$

where $\Lambda$ ia an energy cutoff. For this situation, it is usually considered that a finite expectation value $\Delta_{\Gamma}=$ $\left\langle\psi^{\dagger} \Gamma \psi\right\rangle$ is generated. The magnitude of $\Delta_{\Gamma}$ can be estimated through the energy scale $\Lambda_{U S}$, i.e.,

$$
\Delta_{\Gamma} \sim \Lambda_{U S}=\Lambda e^{-\ell_{c}} .
$$

This method has been usually adopted in the RG studies about the influence of four-fermion interactions on various fermion systems [57 66, 74 77].

If the four-fermion coupling parameter flows to negative infinity finally, we consider that the four-fermion interaction becomes attractive in the low-energy regime. Accordingly, the system is unstable to pairing in the particle-particle channel, namely the generation of superconducting gap.

\section{MEAN FIELD ANALYSIS}

Before performing the RG analysis, in this section, we analyze the generation of various order parameters under the influence of short-range four-fermion interactions through mean field method.

\section{A. Quadratic NLF system}

$$
\text { 1. } \Delta_{1}=\left\langle\psi^{\dagger} \sigma_{1} \psi\right\rangle
$$

Considering $\Delta_{1}$ induced by the four-fermion interaction $\lambda_{1}\left(\psi^{\dagger} \sigma_{1} \psi\right)^{2}$, the fermion propagator in the Matsubara formalism reads as

$$
G\left(\omega_{n}, \mathbf{k}\right)=\frac{1}{-i \omega_{n}+\mathcal{H}_{\mathbf{k}, \Delta_{1}}^{q}},
$$


where

$$
\mathcal{H}_{\mathbf{k}, \Delta_{1}}^{q}=\left[A\left(k_{r}^{2}-k_{z}^{2}\right)+\Delta_{1}\right] \sigma_{1}+2 A k_{r} k_{z} \sigma_{2} .
$$

The mean field equation for $\Delta_{1}$ is given by

$$
\begin{aligned}
\frac{\Delta_{1}}{\lambda_{1}}= & T \sum_{\omega_{n}} \int \frac{d^{3} \mathbf{k}}{(2 \pi)^{3}} \operatorname{Tr}\left[\sigma_{1} G\left(\omega_{n}, \mathbf{k}\right)\right] \\
= & 2 T \int \frac{d^{3} \mathbf{k}}{(2 \pi)^{3}}\left[A\left(k_{r}^{2}-k_{z}^{2}\right)+\Delta_{1}\right] \\
& \times \sum_{\omega_{n}} \frac{1}{\omega_{n}^{2}+E_{\mathbf{k}, \Delta_{1}}^{2}},
\end{aligned}
$$

where $\omega_{n}=(2 n+1) \pi T$ with $n$ being integers, and

$$
E_{\mathbf{k}, \Delta_{1}}=\sqrt{A^{2}\left(k_{r}^{2}+k_{z}^{2}\right)^{2}+2 A\left(k_{r}^{2}-k_{z}^{2}\right) \Delta_{1}+\Delta_{1}^{2}} .
$$

Performing the frequency summation, we get

$$
\begin{aligned}
\Delta_{1}= & \int \frac{d^{3} \mathbf{k}}{(2 \pi)^{3}}\left[A\left(k_{r}^{2}-k_{z}^{2}\right)+\Delta_{1}\right] \frac{1}{E_{\mathbf{k}, \Delta_{1}}} \\
& \times \tanh \left(\frac{E_{\mathbf{k}, \Delta_{1}}}{2 T}\right) .
\end{aligned}
$$

Linearizing $\Delta_{1}$ in the vicinity of critical temperature $T_{c}$ for the phase transition, we obtain the equation for $T_{c}$

$$
\begin{aligned}
\frac{\Delta_{1}}{\lambda_{1}}= & \Delta_{1} \int \frac{d^{3} \mathbf{k}}{(2 \pi)^{3}} \frac{1}{E_{q}(\mathbf{k})} \\
& \times\left\{\left[1-\frac{\left(k_{r}^{2}-k_{z}^{2}\right)^{2}}{\left(k_{r}^{2}+k_{z}^{2}\right)^{2}}\right] \tanh \left(\frac{E_{q}(\mathbf{k})}{2 T_{c}}\right)\right. \\
& \left.+\frac{E_{q}(\mathbf{k})}{2 T_{c}} \frac{1}{\cosh ^{2}\left(\frac{E_{q}(\mathbf{k})}{2 T_{c}}\right)} \frac{\left(k_{r}^{2}-k_{z}^{2}\right)^{2}}{\left(k_{r}^{2}+k_{z}^{2}\right)^{2}}\right\} .
\end{aligned}
$$

The equation can be further written as

$$
\begin{aligned}
\frac{1}{\lambda_{1}}= & \frac{k_{F}}{4 \pi} \int d K K \frac{1}{A K^{2}}\left[\tanh \left(\frac{A K^{2}}{2 T_{c}}\right)\right. \\
& +\frac{A K^{2}}{2 T_{c}} \frac{1}{\left.\cosh ^{2}\left(\frac{A K^{2}}{2 T_{c}}\right)\right]} \\
= & \frac{k_{F}}{8 \pi A}\left[\ln \left(\frac{A \Lambda^{2}}{2 T_{c}}\right) \tanh \left(\frac{A \Lambda^{2}}{2 T_{c}}\right)-\int_{0}^{\frac{A \Lambda^{2}}{2 T_{c}}} d x \ln (x)\right. \\
& \left.\times \frac{1}{\cosh ^{2}(x)}+\tanh \left(\frac{A \Lambda^{2}}{2 T_{c}}\right)\right] .
\end{aligned}
$$

If $T_{c} \ll A \Lambda^{2}$, the equation becomes

$$
\begin{aligned}
\frac{1}{\lambda_{1}} & \approx \frac{k_{F}}{8 \pi A}\left[\ln \left(\frac{A \Lambda^{2}}{2 T_{c}}\right)-\int_{0}^{+\infty} d x \ln (x) \frac{1}{\cosh ^{2}(x)}+1\right] \\
& =\frac{k_{F}}{8 \pi A} \ln \left[\left(\frac{A \Lambda^{2}}{T_{c}}\right)\left(\frac{2 e^{\gamma+1}}{\pi}\right)\right],
\end{aligned}
$$

where $\gamma$ represents the Euler constant, and it satisfies $\gamma \approx 0.577215$. Thus $T_{c}$ is given by

$$
T_{c}=\frac{2 e^{\gamma+1}}{\pi} A \Lambda^{2} e^{-\frac{k_{F}}{8 \lambda_{1} \pi A}} \text {. }
$$

$$
\text { 2. } \Delta_{2}=\left\langle\psi^{\dagger} \sigma_{2} \psi\right\rangle
$$

Incorporating $\Delta_{2}$ induced by the four-fermion interaction $\lambda_{2}\left(\psi^{\dagger} \sigma_{2} \psi\right)^{2}$, after similar derivation shown in subsection IV A 1, we obtain the critical temperature for the phase transition as

$$
\begin{gathered}
T_{c}=\frac{2 e^{\gamma+1}}{\pi} A \Lambda^{2} e^{-\frac{k_{F}}{8 \lambda_{2} \pi A}} . \\
\text { 3. } \Delta_{3}=\left\langle\psi^{\dagger} \sigma_{3} \psi\right\rangle
\end{gathered}
$$

Considering $\Delta_{3}$ induced by the four-fermion interaction $\lambda_{3}\left(\psi^{\dagger} \sigma_{3} \psi\right)^{2}$, the fermion propagator in the Matsubara formalism takes the form

$$
G\left(\omega_{n}, \mathbf{k}\right)=\frac{1}{-i \omega_{n}+\mathcal{H}_{\mathbf{k}, \Delta_{3}}^{q}}
$$

where

$$
\mathcal{H}_{\mathbf{k}, \Delta_{3}}^{q}=A\left[\left(k_{r}^{2}-k_{z}^{2}\right) \sigma_{1}+2 k_{r} k_{z} \sigma_{2}\right]+\Delta_{3} \sigma_{3}
$$

The mean field equation for $\Delta_{3}$ is determined by

$$
\begin{aligned}
\frac{\Delta_{3}}{\lambda_{3}} & =T \sum_{\omega_{n}} \int \frac{d^{3} \mathbf{k}}{(2 \pi)^{3}} \operatorname{Tr}\left[\sigma_{3} G\left(\omega_{n}, \mathbf{k}\right)\right] \\
& =2 \Delta_{3} T \int \frac{d^{3} \mathbf{k}}{(2 \pi)^{3}} \sum_{\omega_{n}} \frac{1}{\omega_{n}^{2}+E_{\mathbf{k}, \Delta_{3}}^{2}},
\end{aligned}
$$

where

$$
E_{\mathbf{k}, \Delta_{3}}=\sqrt{A^{2}\left(k_{r}^{2}+k_{z}^{2}\right)^{2}+\Delta_{3}^{2}} .
$$

Carrying out the frequency summation, we obtain

$$
\frac{\Delta_{3}}{\lambda_{3}}=\Delta_{3} \int \frac{d^{3} \mathbf{k}}{(2 \pi)^{3}} \frac{1}{E_{\mathbf{k}, \Delta_{3}}} \tanh \left(\frac{E_{\mathbf{k}, \Delta_{3}}}{2 T}\right),
$$

which can be further written as

$$
\begin{aligned}
\frac{1}{\lambda_{3}}= & \frac{k_{F}}{2 \pi} \int_{0}^{\Lambda} d K K \frac{1}{\sqrt{A^{2} K^{4}+\Delta_{3}^{2}}} \\
& \times \tanh \left(\frac{\sqrt{A^{2} K^{4}+\Delta_{3}^{2}}}{2 T}\right) .
\end{aligned}
$$

Linearizing $\Delta_{3}$ in the vicinity of $T_{c}$ yields

$$
\begin{aligned}
\frac{1}{\lambda_{3}}= & \frac{k_{F}}{2 \pi} \int_{0}^{\Lambda} d K K \frac{1}{A K^{2}} \tanh \left(\frac{A K^{2}}{2 T_{c}}\right) \\
= & \frac{k_{F}}{4 \pi A}\left[\ln \left(\frac{A \Lambda^{2}}{2 T_{c}}\right) \tanh \left(\frac{A \Lambda^{2}}{2 T_{c}}\right)\right. \\
& \left.-\int_{0}^{\frac{A \Lambda^{2}}{2 T_{c}}} d x \ln (x) \frac{1}{\cosh ^{2}(x)}\right] .
\end{aligned}
$$


If $T_{c} \ll A \Lambda^{2}$, we have

$$
\begin{aligned}
\frac{1}{\lambda_{3}} & \approx \frac{k_{F}}{4 \pi A}\left[\ln \left(\frac{A \Lambda^{2}}{2 T_{c}}\right)-\int_{0}^{+\infty} d x \ln (x) \frac{1}{\cosh ^{2}(x)}\right] \\
& =\frac{k_{F}}{4 \pi A} \ln \left[\left(\frac{A \Lambda^{2}}{T_{c}}\right)\left(\frac{2 e^{\gamma}}{\pi}\right)\right] .
\end{aligned}
$$

Namely

$$
T_{c}=\frac{2 e^{\gamma}}{\pi} A \Lambda^{2} e^{-\frac{k_{F}}{4 \lambda_{3} \pi A}}
$$

\section{B. Cubic NLF system}

$$
\text { 1. } \Delta_{1}=\left\langle\psi^{\dagger} \sigma_{1} \psi\right\rangle
$$

Considering $\Delta_{1}$ generated by the four-fermion interaction $\lambda_{1}\left(\psi^{\dagger} \sigma_{1} \psi\right)^{2}$, the fermion propagator in Matsubara formalism can be written as

$$
G\left(\omega_{n}, \mathbf{k}\right)=\frac{1}{-i \omega_{n}+\mathcal{H}_{\mathbf{k}, \Delta_{1}}^{c}},
$$

where

$$
\begin{aligned}
\mathcal{H}_{\mathbf{k}, \Delta_{1}}^{c}= & {\left[B\left(k_{r}^{3}-3 k_{r} k_{z}^{2}\right)+\Delta_{1}\right] \sigma_{1} } \\
& +B\left(k_{z}^{3}-3 k_{z} k_{r}^{2}\right) \sigma_{2} .
\end{aligned}
$$

The mean field equation for $\Delta_{1}$ takes the form

$$
\begin{aligned}
\frac{\Delta_{1}}{\lambda_{1}}= & T \sum_{\omega_{n}} \int \frac{d^{3} \mathbf{k}}{(2 \pi)^{3}} \operatorname{Tr}\left[\sigma_{1} G\left(\omega_{n}, \mathbf{k}\right)\right] \\
= & 2 T \int \frac{d^{3} \mathbf{k}}{(2 \pi)^{3}}\left[B\left(k_{r}^{3}-3 k_{r} k_{z}^{2}\right)+\Delta_{1}\right] \\
& \times \sum_{\omega_{n}} \frac{1}{\omega_{n}^{2}+E_{\mathbf{k}, \Delta_{1}}^{2}},
\end{aligned}
$$

where

$$
E_{\mathbf{k}, \Delta_{1}}=\sqrt{B^{2}\left(k_{r}^{2}+k_{z}^{2}\right)^{3}+2 B\left(k_{r}^{3}-3 k_{r} k_{z}^{2}\right) \Delta_{1}+\Delta_{1}^{2}} .
$$

Carrying out the frequency summation gives to

$$
\begin{aligned}
\frac{\Delta_{1}}{\lambda_{1}}= & \int \frac{d^{3} \mathbf{k}}{(2 \pi)^{3}}\left[B\left(k_{r}^{3}-3 k_{r} k_{z}^{2}\right)+\Delta_{1}\right] \frac{1}{E_{\mathbf{k}, \Delta_{1}}} \\
& \times \tanh \left(\frac{E_{\mathbf{k}, \Delta_{1}}}{2 T}\right) .
\end{aligned}
$$

Linearizing $\Delta_{1}$ in the vicinity of $T_{c}$, we find

$$
\begin{aligned}
\frac{\Delta_{1}}{\lambda_{1}}= & \Delta_{1} \int \frac{d^{3} \mathbf{k}}{(2 \pi)^{3}} \frac{1}{E_{c}(\mathbf{k})} \\
& \times\left\{\left[1-\frac{\left(k_{r}^{3}-3 k_{r} k_{z}^{2}\right)^{2}}{\left(k_{r}^{2}+k_{z}^{2}\right)^{3}}\right] \tanh \left(\frac{E_{c}(\mathbf{k})}{2 T_{c}}\right)\right. \\
& \left.+\frac{E_{c}(\mathbf{k})}{2 T_{c}} \frac{1}{\cosh ^{2}\left(\frac{E_{c}(\mathbf{k})}{2 T_{c}}\right)} \frac{\left(k_{r}^{3}-3 k_{r} k_{z}^{2}\right)^{2}}{\left(k_{r}^{2}+k_{z}^{2}\right)^{3}}\right\} .
\end{aligned}
$$

This equation can be further expressed as

$$
\begin{aligned}
\frac{1}{\lambda_{1}}= & \frac{k_{F}}{4 \pi} \int_{0}^{\Lambda} d K K \frac{1}{B K^{3}} \tanh \left(\frac{B K^{3}}{2 T_{c}}\right) \\
= & \frac{k_{F}}{12 \pi B^{2 / 3}\left(2 T_{c}\right)^{1 / 3}}\left[(-3) \frac{1}{\left(\frac{B \Lambda^{3}}{2 T_{c}}\right)^{1 / 3}} \tanh \left(\frac{B \Lambda^{3}}{2 T_{c}}\right)\right. \\
& \left.+4 \int_{0}^{\frac{B \Lambda^{3}}{2 T_{c}}} d x \frac{1}{x^{1 / 3}} \frac{1}{\cosh ^{2}(x)}\right] .
\end{aligned}
$$

In the limit $T_{c} \ll B \Lambda^{3}$, we get

$$
\begin{aligned}
\frac{1}{\lambda_{1}} & \approx \frac{k_{F}}{12 \pi B^{2 / 3}\left(2 T_{c}\right)^{1 / 3}} 4 \int_{0}^{+\infty} d x \frac{1}{x^{1 / 3}} \frac{1}{\cosh ^{2}(x)} \\
& =\frac{a k_{F}}{3 \cdot 2^{1 / 3} \pi B^{2 / 3} T_{c}^{1 / 3}}
\end{aligned}
$$

where

$$
a=\int_{0}^{+\infty} d x \frac{1}{x^{1 / 3}} \frac{1}{\cosh ^{2}(x)} \approx 1.43829 .
$$

Accordingly, $T_{c}$ can be expressed as

$$
T_{c}=\left(\frac{a \lambda_{1} k_{F}}{3 \cdot 2^{1 / 3} \pi B^{2 / 3}}\right)^{3} .
$$

$$
\text { 2. } \Delta_{2}=\left\langle\psi^{\dagger} \sigma_{2} \psi\right\rangle
$$

Considering $\Delta_{2}$ induced by the four-fermion interaction $\lambda_{2}\left(\psi^{\dagger} \sigma_{2} \psi\right)^{2}$, similar to subsection IVB 1, we obtain

$$
T_{c}=\left(\frac{a \lambda_{2} k_{F}}{3 \cdot 2^{1 / 3} \pi B^{2 / 3}}\right)^{3} .
$$

$$
\text { 3. } \Delta_{3}=\left\langle\psi^{\dagger} \sigma_{3} \psi\right\rangle
$$

Incorporating $\Delta_{3}$ induced by the four-fermion interaction $\lambda_{3}\left(\psi^{\dagger} \sigma_{3} \psi\right)^{2}$, the fermion propagator in the Matsubara formalism reads as

$$
G\left(\omega_{n}, \mathbf{k}\right)=\frac{1}{-i \omega_{n}+\mathcal{H}_{\mathbf{k}, \Delta_{3}}^{c}},
$$

where

$$
\begin{aligned}
\mathcal{H}_{\mathbf{k}, \Delta_{3}}^{c}= & B\left[\left(k_{r}^{3}-3 k_{r} k_{z}^{2}\right) \sigma_{1}+\left(k_{z}^{3}-3 k_{z} k_{r}^{2}\right) \sigma_{2}\right] \\
& +\Delta_{3} \sigma_{3} .
\end{aligned}
$$

The mean field equation for $\Delta_{3}$ is given by

$$
\begin{aligned}
\frac{\Delta_{3}}{\lambda_{3}} & =T \sum_{\omega_{n}} \int \frac{d^{3} \mathbf{k}}{(2 \pi)^{3}} \operatorname{Tr}\left[\sigma_{3} G_{0}\left(\omega_{n}, \mathbf{k}\right)\right] \\
& =2 \Delta_{3} T \int \frac{d^{3} \mathbf{k}}{(2 \pi)^{3}} \sum_{\omega_{n}} \frac{1}{\omega_{n}^{2}+E_{\mathbf{k}, \Delta_{3}}^{2}},
\end{aligned}
$$


where

$$
E_{\mathbf{k}, \Delta_{3}}=\sqrt{B^{2}\left(k_{r}^{2}+k_{z}^{2}\right)^{3}+\Delta_{3}^{2}}
$$

Performing the frequency summation, we get

$$
\frac{\Delta_{3}}{\lambda_{3}}=\Delta_{3} \int \frac{d^{3} \mathbf{k}}{(2 \pi)^{3}} \frac{1}{E_{\mathbf{k}, \Delta_{3}}} \tanh \left(\frac{E_{\mathbf{k}, \Delta_{3}}}{2 T}\right) .
$$

The equation can be further written as

$$
\begin{aligned}
\frac{1}{\lambda_{3}}= & \frac{k_{F}}{2 \pi} \int_{0}^{\Lambda} d K K \frac{1}{\sqrt{B^{2} K^{6}+\Delta_{3}^{2}}} \\
& \times \tanh \left(\frac{\sqrt{B^{2} K^{6}+\Delta_{3}^{2}}}{2 T}\right) .
\end{aligned}
$$

$T_{c}$ is determined by

$$
\begin{aligned}
\frac{1}{\lambda_{3}}= & \frac{k_{F}}{2 \pi} \int_{0}^{\Lambda} d K K \frac{1}{B K^{3}} \tanh \left(\frac{B K^{3}}{2 T_{c}}\right) \\
= & \frac{k_{F}}{6 \pi B^{2 / 3}\left(2 T_{c}\right)^{1 / 3}}\left[(-3) \frac{1}{\left(\frac{B \Lambda^{3}}{2 T_{c}}\right)^{1 / 3}} \tanh \left(\frac{B \Lambda^{3}}{2 T_{c}}\right)\right. \\
& \left.+3 \int_{0}^{\frac{B \Lambda^{3}}{2 T_{c}}} d x \frac{1}{x^{1 / 3}} \frac{1}{\cosh ^{2}(x)}\right]
\end{aligned}
$$

In the limit $T_{c} \ll B \Lambda^{3}$, we obtain

$$
\begin{aligned}
\frac{1}{\lambda_{3}} & \approx \frac{k_{F}}{6 \pi B^{2 / 3}\left(2 T_{c}\right)^{1 / 3}} 3 \int_{0}^{+\infty} d x \frac{1}{x^{1 / 3}} \frac{1}{\cosh ^{2}(x)} \\
& =\frac{a k_{F}}{2^{4 / 3} \pi B^{2 / 3} T_{c}^{1 / 3}},
\end{aligned}
$$

which is equivalent to

$$
T_{c}=\left(\frac{a \lambda_{3} k_{F}}{2^{4 / 3} \pi B^{2 / 3}}\right)^{3} .
$$

\section{RENORMALIZATION GROUP ANALYSIS}

In this section, we present the RG results of the influence of four-fermion interactions on quadratic and cubic NLF systems. The detailed derivation for the RG equations are shown in the Appendices.

\section{A. Quadratic NLF}

For quadratic NLF system in the presence of fourfermion interactions, the RG equations for the coupling
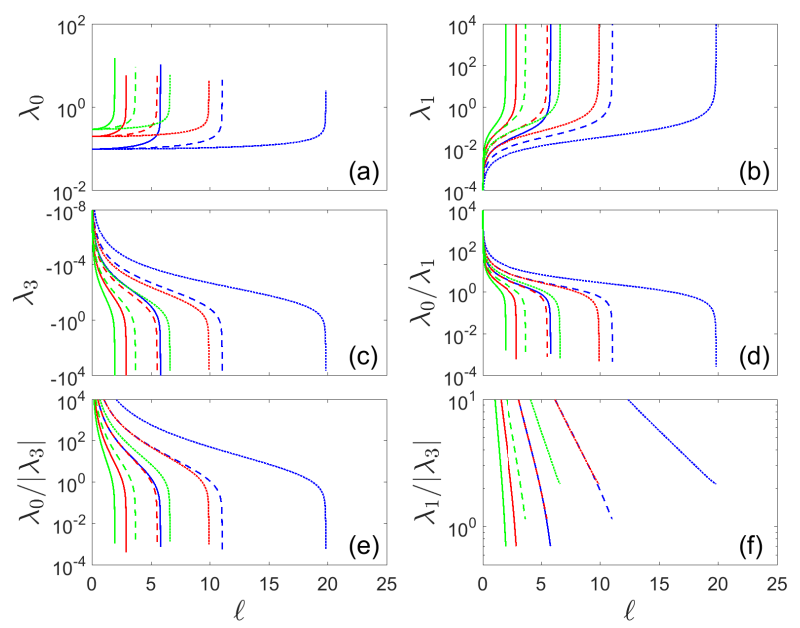

FIG. 2: Flows of $\lambda_{0}, \lambda_{1}, \lambda_{3}, \lambda_{0} / \lambda_{1}, \lambda_{0} /\left|\lambda_{3}\right|$, and $\lambda_{1} /\left|\lambda_{3}\right|$ in quadratic NLF system. Blue, red, and green colors correspond to $\lambda_{0,0}=0.1,0.2,0.3$ respectively. Solid, dashed, and dotted lines stand for $N=1,2,4$ respectively.

parameters are given by

$$
\begin{aligned}
\frac{d \lambda_{0}}{d \ell}= & \left(\lambda_{0} \lambda_{1}+\lambda_{0} \lambda_{2}\right) \frac{1}{N} \\
\frac{d \lambda_{1}}{d \ell}= & {\left[\left(\lambda_{0}^{2}+\lambda_{2}^{2}+\lambda_{3}^{2}+\lambda_{0} \lambda_{1}+2 \lambda_{1} \lambda_{2}+\lambda_{1} \lambda_{3}\right.\right.} \\
& \left.\left.-2 \lambda_{2} \lambda_{3}\right) \frac{1}{N}+\lambda_{1}^{2}\right] \\
\frac{d \lambda_{2}}{d \ell}= & {\left[\left(\lambda_{0}^{2}+\lambda_{1}^{2}+\lambda_{3}^{2}+\lambda_{0} \lambda_{2}+2 \lambda_{1} \lambda_{2}-2 \lambda_{1} \lambda_{3}\right.\right.} \\
& \left.\left.+\lambda_{2} \lambda_{3}\right) \frac{1}{N}+\lambda_{2}^{2}\right] \\
\frac{d \lambda_{3}}{d \ell}= & {\left[\left(-2 \lambda_{3}^{2}+2 \lambda_{0} \lambda_{3}-2 \lambda_{1} \lambda_{2}+3 \lambda_{1} \lambda_{3}+3 \lambda_{2} \lambda_{3}\right)\right.} \\
& \left.\times \frac{1}{N}+2 \lambda_{3}^{2}\right]
\end{aligned}
$$

The transformations $\frac{k_{F}}{2 \pi A} \lambda_{i} \rightarrow \lambda_{i}$ with $i=0,1,2,3$ have been employed in the derivation of the RG equations. We notice that $\lambda_{0}$ is not generated if the initial value $\lambda_{0,0}=0$.

If the initial value $\lambda_{0,0}$ is finite, the flows of $\lambda_{0}, \lambda_{1}, \lambda_{3}$, $\lambda_{0} / \lambda_{1}, \lambda_{0} /\left|\lambda_{3}\right|, \lambda_{1} /\left|\lambda_{3}\right|$ are shown in Fig. 2, As shown in Figs. 2(a)-2(c), $\lambda_{0}, \lambda_{1}$ approach to infinity, and $\lambda_{3}$ flows to negative infinity at same finite energy scale. According to Figs. 2(d)-2(f), $\lambda_{0} / \lambda_{1}$ and $\lambda_{0} /\left|\lambda_{3}\right|$ approach to zero. Additionally, $\lambda_{1} /\left|\lambda_{3}\right|$ flows to a constant smaller than 1 for $N=1$, but approaches to a constant larger than 1 for $N \geq 2$. $\lambda_{1} / \lambda_{2}$ is always equal to 1 , which is not shown in Fig. 2. These results indicate that arbitrarily weak four-fermion interaction induces the system to be unstable. For $N=1$, generation of superconducting gap is the leading instability. However, splitting of quadratic nodal line with generation of $\Delta_{1}$ or $\Delta_{2}$ is the subleading instability. For $N \geq 2$, splitting of quadratic nodal line with 

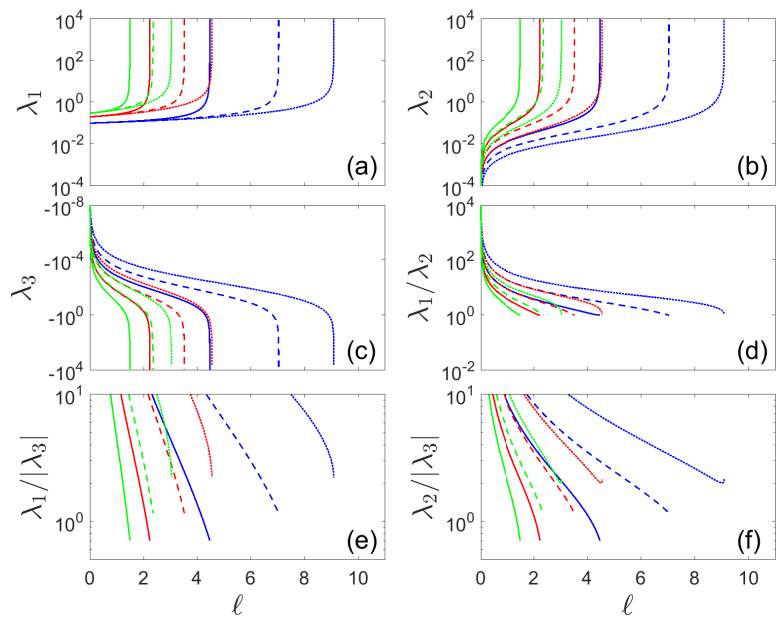

FIG. 3: Flows of $\lambda_{1}, \lambda_{2}, \lambda_{3}, \lambda_{1} / \lambda_{2}, \lambda_{1} /\left|\lambda_{3}\right|$, and $\lambda_{2} /\left|\lambda_{3}\right|$ in quadratic NLF system. Blue, red, and green colors correspond to $\lambda_{1,0}=0.1,0.2,0.3$ respectively. Solid, dashed, and dotted lines stand for $N=1,2,4$ respectively.

generation of $\Delta_{1}$ or $\Delta_{2}$ becomes the leading instability.

If the parameter $\lambda_{1,0}$ takes finite value, the relations between $\lambda_{1}, \lambda_{2}, \lambda_{3}, \lambda_{1} / \lambda_{2}, \lambda_{1} /\left|\lambda_{3}\right|, \lambda_{2} /\left|\lambda_{3}\right|$ and running parameter $\ell$ are shown in Fig. 3. $\lambda_{0}$ always equals to zero, if only $\lambda_{1,0}$ is finite initially. We can find that $\lambda_{1}$ always approaches to infinity at some finite value $\ell_{c}$. As shown in Fig. 3(b), $\lambda_{2}$ flows from zero to infinity at the same $\ell_{c}$. According to Fig. 3(c), $\lambda_{3}$ is generated from zero and approaches to negative infinity finally. As depicted in Figs. [3(d)-33(f), $\lambda_{1} / \lambda_{2}$ flows to 1 , and $\lambda_{1} /\left|\lambda_{3}\right|$ and $\lambda_{2} /\left|\lambda_{3}\right|$ flow to a constant smaller than 1 for $N=1$ but flow to a constant larger than 1 for $N \geq 2$. These results represent that transition into superconducting phase is the leading instability for $N=1$, but generation of $\Delta_{1}$ or $\Delta_{2}$ and splitting of quadratic nodal line into conventional nodal lines is the leading instability for $N \geq 2$.

If the parameter $\lambda_{2,0}$ takes finite value, we will obtain qualitatively similar results comparing the ones in the case that only $\lambda_{1,0}$ is finite.

If the initial value $\lambda_{3,0}$ is finite, the flows of $\lambda_{1}, \lambda_{2}, \lambda_{3}$, $\lambda_{1} / \lambda_{2}, \lambda_{1} / \lambda_{3}, \lambda_{2} / \lambda_{3}$ are shown in Fig. 14. According to Figs. 4(a)-4(c), $\lambda_{3}$ approaches to infinity at a finite $\ell_{c}$, and $\lambda_{1}$ and $\lambda_{2}$ flow from zero and approach to infinity at the same $\ell_{c}$. As shown in Fig. 4 (d), $\lambda_{1} / \lambda_{2}$ equals to

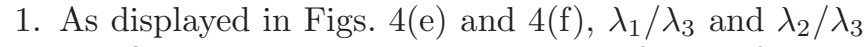
always flow to a constant smaller than 1 for any fermion flavor $N$. It represents that generation of excitonic gap is always the leading instability for any fermion flavor, if only $\lambda_{3,0}$ takes finite value.

If the initial values of two coupling parameters are finite, the flows of $\lambda_{0}, \lambda_{1}, \lambda_{2}, \lambda_{3}, \lambda_{0} /\left|\lambda_{3}\right|, \lambda_{1} / \lambda_{2}, \lambda_{1} /\left|\lambda_{3}\right|$, $\lambda_{2} /\left|\lambda_{3}\right|$ are displayed in Fig. 5. According to these results, we find that the system could be driven to NLSM, excitonic insulator, or superconducting phase, which is determined by the concrete initial conditions and fermion flavor sensitively.
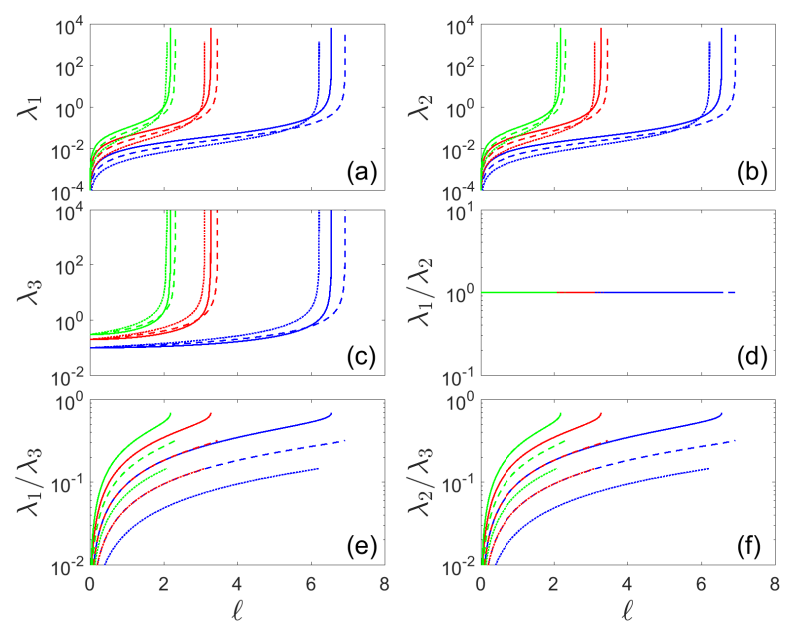

FIG. 4: Flows of $\lambda_{1}, \lambda_{2}, \lambda_{3}, \lambda_{1} / \lambda_{2}, \lambda_{1} / \lambda_{3}$, and $\lambda_{2} / \lambda_{3}$ in quadratic NLF system. Blue, red, and green colors correspond to $\lambda_{3,0}=0.1,0.2,0.3$ respectively. Solid, dashed, and dotted lines stand for $N=1,2,4$ respectively.

\section{B. Cubic NLF}

For cubic NLF system in the presence of four-fermion interactions, the RG equations for the four-fermion coupling parameters are given by

$$
\begin{aligned}
\frac{d \lambda_{0}}{d \ell}= & \frac{1}{3} \lambda_{0}+\left(\lambda_{1} \lambda_{3}+\lambda_{2} \lambda_{3}\right) \frac{1}{N}, \\
\frac{d \lambda_{1}}{d \ell}= & \frac{1}{3} \lambda_{1}+\left[\left(-\lambda_{1}^{2}+\lambda_{0} \lambda_{1}+\lambda_{0} \lambda_{3}+\lambda_{1} \lambda_{2}+\lambda_{1} \lambda_{3}\right.\right. \\
& \left.\left.-2 \lambda_{2} \lambda_{3}\right) \frac{1}{N}+\lambda_{1}^{2}\right], \\
\frac{d \lambda_{2}}{d \ell}= & \frac{1}{3} \lambda_{2}+\left[\left(-\lambda_{2}^{2}+\lambda_{0} \lambda_{2}+\lambda_{0} \lambda_{3}+\lambda_{1} \lambda_{2}-2 \lambda_{1} \lambda_{3}\right.\right. \\
& \left.\left.+\lambda_{2} \lambda_{3}\right) \frac{1}{N}+\lambda_{2}^{2}\right], \\
\frac{d \lambda_{3}}{d \ell}= & \frac{1}{3} \lambda_{3}+\left[\left(-2 \lambda_{3}^{2}+\lambda_{0} \lambda_{1}+\lambda_{0} \lambda_{2}+2 \lambda_{0} \lambda_{3}-2 \lambda_{1} \lambda_{2}\right.\right. \\
& \left.\left.+2 \lambda_{1} \lambda_{3}+2 \lambda_{2} \lambda_{3}\right) \frac{1}{N}+2 \lambda_{3}^{2}\right] .
\end{aligned}
$$

The transformations $\frac{k_{F}}{2 \pi B \Lambda} \lambda_{i} \rightarrow \lambda_{i}$ with $i=0,1,2,3$ have been utilized. One could find that one type of fourfermion interaction can exist solely.

If only consider the four-fermion interaction $\lambda_{0}\left(\psi^{\dagger} \sigma_{0} \psi\right)^{2}$, RG equation for the coupling strength takes the form

$$
\frac{d \lambda_{0}}{d \ell}=\frac{1}{3} \lambda_{0}
$$

The solution is

$$
\lambda_{0}=\lambda_{0,0} e^{\frac{1}{3} \ell} .
$$

It is easy to find that $\lambda_{0}$ does not become divergent at a finite energy scale, but only becomes divergent in the 

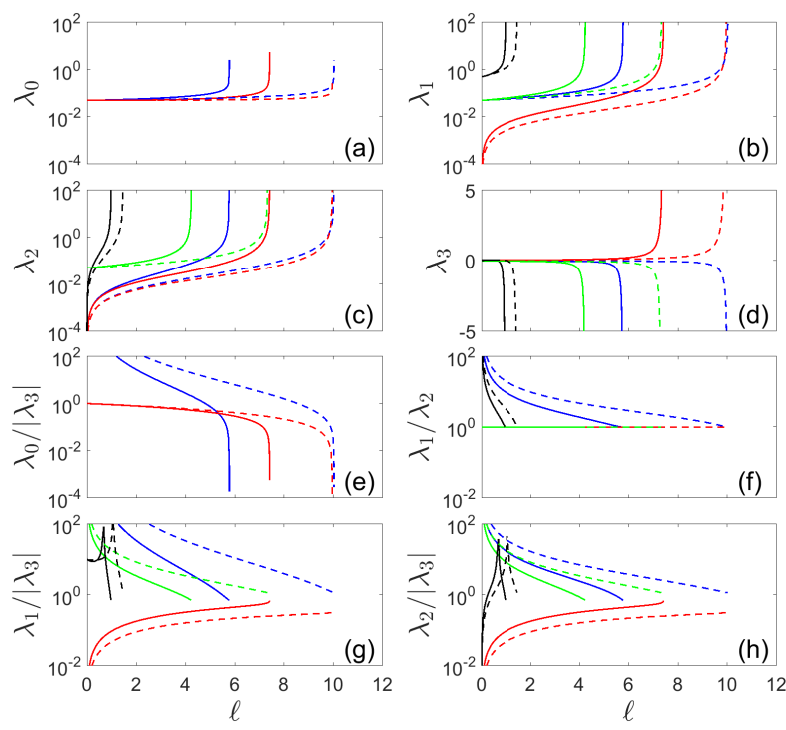

FIG. 5: Flows of $\lambda_{0}, \lambda_{1}, \lambda_{2}, \lambda_{3} \lambda_{0} /\left|\lambda_{3}\right|, \lambda_{1} / \lambda_{2}, \lambda_{1} /\left|\lambda_{3}\right|$, and $\lambda_{2} /\left|\lambda_{3}\right|$ in quadratic NLF system. Blue color corresponds to $\lambda_{0,0}=0.05$ and $\lambda_{1,0}=0.05$; Red color corresponds to $\lambda_{0,0}=$ 0.05 and $\lambda_{3,0}=0.05$; Green color corresponds to $\lambda_{1,0}=0.05$ and $\lambda_{2,0}=0.05$; Black color corresponds to $\lambda_{1,0}=0.05$ and $\lambda_{3,0}=0.05$; Solid and dashed lines stand for $N=1$ and $N=2$ respectively.

lowest energy limit $\ell \rightarrow \infty$, i.e, $\ell_{c} \rightarrow \infty$. We believe that divergence of four-fermion coupling strength at $\ell \rightarrow \infty$ does not represent the generation of a finite expectation value of order parameter. Indeed, according to Eqs. (21) and (22), the energy scale for the appearance of instability and the magnitude of order parameter vanish if $\ell_{c} \rightarrow \infty$.

If only the four-fermion interaction $\lambda_{1}\left(\psi^{\dagger} \sigma_{1} \psi\right)^{2}$ is considered, the RG equation for $\lambda_{1}$ is given by

$$
\frac{d \lambda_{1}}{d \ell}=\frac{1}{3} \lambda_{1}+\left(1-\frac{1}{N}\right) \lambda_{1}^{2} .
$$

For the fermion flavor $N=1$, the RG equation becomes

$$
\frac{d \lambda_{1}}{d \ell}=\frac{1}{3} \lambda_{1}
$$

The corresponding solution reads as

$$
\lambda_{1}=\lambda_{1,0} e^{\frac{1}{3} \ell},
$$

which becomes divergent in the lowest energy limit $\ell \rightarrow$ $\infty$. In this case, divergence of $\lambda_{1}$ does not indicate the generation of long-range order parameter $\Delta_{1}=\left\langle\psi^{\dagger} \sigma_{1} \psi\right\rangle$.

For the case $N>1$, solving Eq. (722) yields

$$
\lambda_{1}=\frac{\frac{1}{3} \lambda_{1,0} e^{\frac{1}{3} \ell}}{\frac{1}{3}+\left(1-\frac{1}{N}\right) \lambda_{1,0}\left(1-e^{\frac{1}{3} \ell}\right)} .
$$

We find that $\lambda_{1}$ becomes divergent at a critical value

$$
\ell_{1 c}=3 \ln \left[1+\frac{1}{3\left(1-\frac{1}{N}\right) \lambda_{1,0}}\right] .
$$
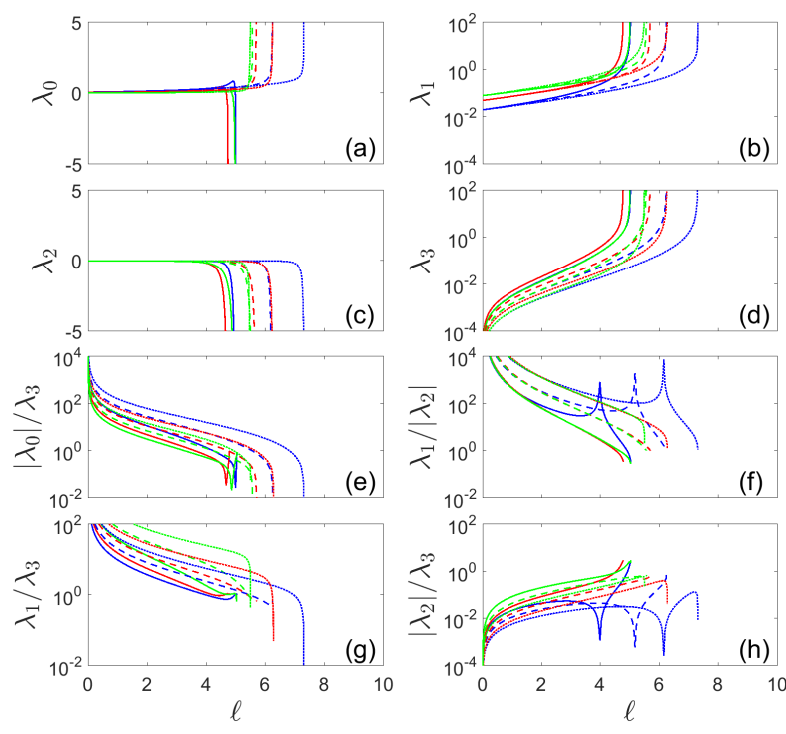

FIG. 6: Flows of $\lambda_{0}, \lambda_{1}, \lambda_{2}, \lambda_{3},\left|\lambda_{0}\right| / \lambda_{3}, \lambda_{1} /\left|\lambda_{2}\right|, \lambda_{1} / \lambda_{3}$ and $\left|\lambda_{2}\right| / \lambda_{3}$ in cubic NLF system. Blue color corresponds to $\lambda_{0,0}=$ 0.08 and $\lambda_{1,0}=0.02 ;$ Red color corresponds to $\lambda_{0,0}=0.05$ and $\lambda_{1,0}=0.05$; Green color corresponds to $\lambda_{0,0}=0.02$ and $\lambda_{1,0}=0.08$. Solid, dashed, and dotted lines stand for $N=$ $1,2,4$ respectively.

This result implies that a finite expectation value $\Delta_{1}=$ $\left\langle\psi^{\dagger} \sigma_{1} \psi\right\rangle$ is generated under the influence of four-fermion interaction. The magnitude of $\Delta_{1}$ can be roughly estimated by

$$
\Delta_{1} \sim \Lambda e^{-\ell_{1 c}},
$$

where $\Lambda$ is an energy cutoff.

Similarly, if only the four-fermion interaction $\lambda_{2}\left(\psi^{\dagger} \sigma_{2} \psi\right)^{2}$ is considered, we could find that a finite expectation value $\Delta_{2}=\left\langle\psi^{\dagger} \sigma_{2} \psi\right\rangle$ is generated for $N>1$.

Considering only the four-fermion interaction $\lambda_{3}\left(\psi^{\dagger} \sigma_{3} \psi\right)^{2}$, the RG equation for the coupling strength can be written as

$$
\frac{d \lambda_{3}}{d \ell}=\frac{1}{3} \lambda_{3}+2\left(1-\frac{1}{N}\right) \lambda_{3}^{2} .
$$

If $N=1$, the RG equation becomes

$$
\frac{d \lambda_{3}}{d \ell}=\frac{1}{3} \lambda_{3}
$$

The corresponding solution is given by

$$
\lambda_{3}=\lambda_{3,0} e^{\frac{1}{3} \ell},
$$

which is divergent in the limit $\ell \rightarrow \infty$. Thus, for $N=1$, finite expectation value $\Delta_{3}=\left\langle\psi^{\dagger} \sigma_{3} \psi\right\rangle$ should not be generated.

For $N>1$, solving Eq. (178) gives rise to

$$
\lambda_{3}=\frac{\frac{1}{3} \lambda_{3,0} e^{\frac{1}{3} \ell}}{\frac{1}{3}+2\left(1-\frac{1}{N}\right) \lambda_{3,0}\left(1-e^{\frac{1}{3} \ell}\right)},
$$



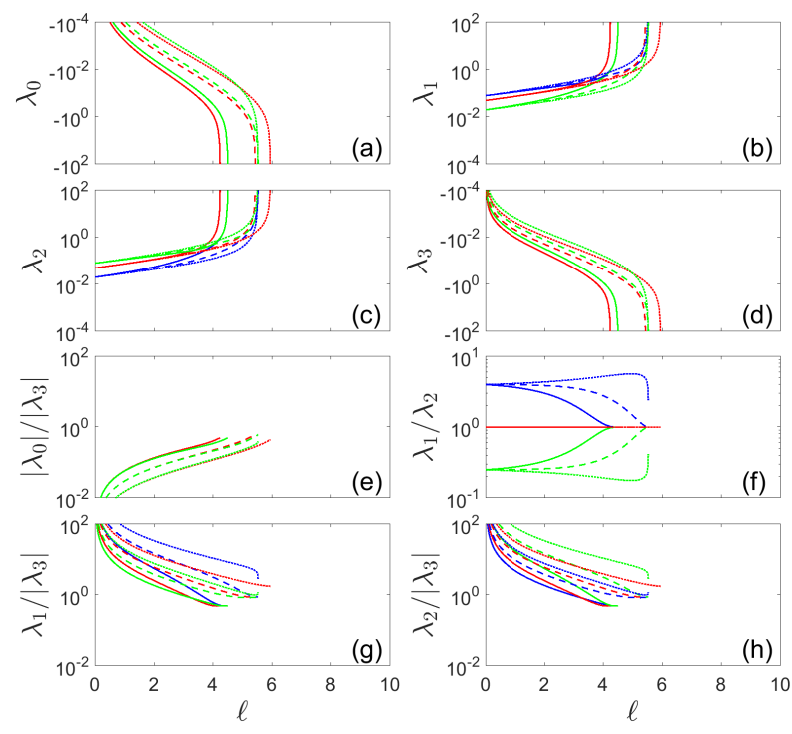

FIG. 7: Flows of $\lambda_{0}, \lambda_{1}, \lambda_{2}, \lambda_{3},\left|\lambda_{0}\right| /\left|\lambda_{3}\right| \lambda_{1} / \lambda_{2}, \lambda_{1} /\left|\lambda_{3}\right|$, and $\lambda_{2} /\left|\lambda_{3}\right|$ in cubic NLF system. Blue color corresponds to $\lambda_{1,0}=0.08$ and $\lambda_{2,0}=0.02$; Red color corresponds to $\lambda_{1,0}=$ 0.05 and $\lambda_{2,0}=0.05$; Green color corresponds to $\lambda_{1,0}=0.02$ and $\lambda_{2,0}=0.08$. Solid, dashed, and dotted lines stand for $N=1,2,4$ respectively.

It is found that $\lambda_{3}$ approaches to infinity when $\ell \rightarrow \ell_{3 c}$, where

$$
\ell_{3 c}=3 \ln \left[1+\frac{1}{6\left(1-\frac{1}{N}\right) \lambda_{3,0}}\right] .
$$

Therefore, for $N>1$, finite expectation value $\Delta_{3}$ should be generated under the influence of four-fermion interaction $\lambda_{3}\left(\psi^{\dagger} \sigma_{3} \psi\right)^{2}$. The magnitude of $\Delta_{3}$ can be estimated by

$$
\Delta_{3} \sim \Lambda e^{-\ell_{3 c}} .
$$

A shown in above, if one type of four-fermion coupling is considered and $N=1$, long-range order is not generated in cubic NLF system. Accordingly, the DOS takes the behavior $\rho(\omega) \sim \omega^{-1 / 3}$ which is divergent in the limit $\omega \rightarrow 0$, and compressibility takes the behavior $C_{v}(T) \sim T^{-1 / 3}$ which is divergent in the limit $T \rightarrow 0$, as shown in the Sec.VI. We notice that these characteristics are similar to ones in supermetal state proposed by Isobe and $\mathrm{Fu}[78]$. Indeed, as shown in the paper by Isobe and $\mathrm{Fu}$, in the supermetal state, none long-range order appears, DOS becomes divergent in the limit $\omega \rightarrow 0$, and compressibility becomes divergent in the limit $T \rightarrow 0$.

If the initial values of two coupling parameters are finite, the flows of $\lambda_{0}, \lambda_{1}, \lambda_{2}, \lambda_{3}$, and the ratios between them are shown in Figs. 6.8. We notice that the coupling parameters which vanish initially are generated. The absolute values of $\lambda_{0}, \lambda_{1}, \lambda_{2}$, and $\lambda_{3}$ all approach to infinity at a finite RG running parameter $\ell_{c}$. The ratios between the coupling parameters in the limit $\ell \rightarrow \ell_{c}$ are deter-
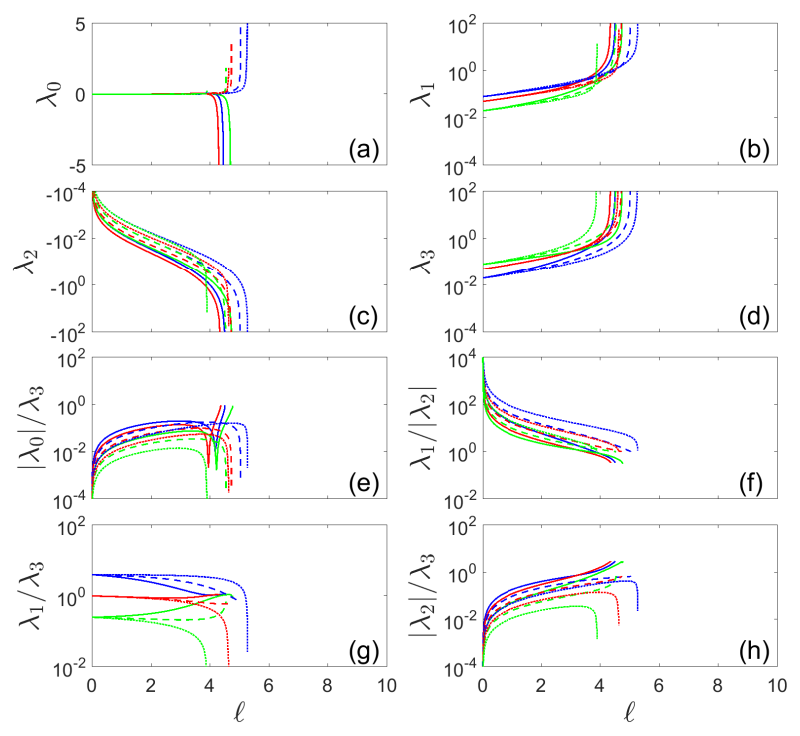

FIG. 8: Flows of $\lambda_{0}, \lambda_{1}, \lambda_{2}, \lambda_{3},\left|\lambda_{0}\right| / \lambda_{3}, \lambda_{1} /\left|\lambda_{2}\right|, \lambda_{1} / \lambda_{3}$, and $\left|\lambda_{2}\right| / \lambda_{3}$ in cubic NLF system. Blue color corresponds to $\lambda_{1,0}=0.08$ and $\lambda_{3,0}=0.02$; Red color corresponds to $\lambda_{1,0}=$ 0.05 and $\lambda_{3,0}=0.05$; Green color corresponds to $\lambda_{1,0}=0.02$ and $\lambda_{3,0}=0.08$. Solid, dashed, and dotted lines stand for $N=1,2,4$ respectively.

mined by the initial conditions. After checking these ratios, we find that the system would become to NLSM, excitonic insulator, or superconducting phase, according to the concrete initial conditions and the value of $N$.

\section{OBSERVABLE QUANTITIES}

For convenience, we compare the observable quantities in different phases.

For conventional NLF system, the DOS satisfies

$$
\rho(\omega)=\frac{N k_{F}|\omega|}{2 \pi v_{F} v_{z}},
$$

where $v_{F}$ and $v_{z}$ are the fermion velocities within the $x-y$ plane and along the $z$ axis. The specific heat and compressibility depend on temperature as

$$
\begin{aligned}
C_{v}(T) & =\frac{9 \zeta(3) N k_{F}}{\pi v_{F} v_{z}} T^{2}, \\
\kappa(T) & =\frac{2 \ln (2) N k_{F}}{\pi v_{F} v_{z}} T,
\end{aligned}
$$

where $\zeta(x)$ is the Riemann zeta function.

For quadratic and cubic NLF systems with an excitonic gap $\Delta_{3}$, the retarded fermion propagator takes the form

$$
G_{q, c}^{\mathrm{ret}}(\omega, \mathbf{k})=\frac{1}{-(\omega+i \eta)+\mathcal{H}_{0}^{q, c}(\mathbf{k})+\Delta_{3} \sigma_{3}},
$$

where $\eta$ is infinitesimal. The spectral function is given 
by

$$
\begin{aligned}
\mathcal{A}_{q, c}(\omega, \mathbf{k}) & =\frac{1}{\pi} \operatorname{Tr}\left[\operatorname{Im}\left[G_{q, c}^{\mathrm{ret}}(\omega, \mathbf{k})\right]\right] \\
& =2|\omega| \delta\left(\omega^{2}-\left(E_{q, c}^{2}(\mathbf{k})+\Delta_{3}^{2}\right)\right) .
\end{aligned}
$$

The DOS can be written as

$$
\begin{aligned}
\rho_{q, c}(\omega) & =N \int \frac{d^{3} \mathbf{k}}{(2 \pi)^{3}} \mathcal{A}_{q, c}(\omega, \mathbf{k}) \\
& \approx N k_{F} \int \frac{d k_{r} d k_{z}}{(2 \pi)^{2}} \mathcal{A}_{q, c}(\omega, \mathbf{k}) .
\end{aligned}
$$

Substituting Eq. (21) into Eqs. (88) and (89), we can get the DOS for quadratic NLF system

$$
\rho_{q}(\omega)=\frac{N k_{F}|\omega|}{4 \pi A \sqrt{\omega^{2}-\Delta_{3}^{2}}} \theta\left(|\omega|-\left|\Delta_{3}\right|\right),
$$

where $\theta(x)$ represents the Heaviside step function. Substituting Eq. (4) into Eqs. (88) and (89), the DOS for cubic NLF system can be written as

$$
\rho_{c}(\omega)=\frac{N k_{F}|\omega|}{6 \pi B^{2 / 3}\left(\omega^{2}-\Delta_{3}^{2}\right)^{2 / 3}} \theta\left(|\omega|-\left|\Delta_{3}\right|\right) .
$$

If $\Delta_{3}=0, \rho_{q}$ and $\rho_{c}$ become

$$
\rho_{q}(\omega)=\frac{N k_{F}}{4 \pi A},
$$

and

$$
\rho_{c}(\omega)=\frac{N k_{F}}{6 \pi B^{2 / 3}|\omega|^{1 / 3}},
$$

respectively.

For quadratic and cubic NLF systems with finite excitonic gap $\Delta_{3}$ and finite chemical potential $\mu$, the propagator of fermions in the Matsubara formalism can be written as

$$
\begin{aligned}
G_{q, c}\left(\omega_{n}, \mathbf{k}\right) & =\frac{1}{-\left(i \omega_{n}+\mu\right)+\mathcal{H}_{0}^{q, c}(\mathbf{k})+\Delta_{3} \sigma_{3}} \\
& =\frac{i \omega_{n}+\mu+\mathcal{H}_{0}^{q, c}(\mathbf{k})+\Delta_{3} \sigma_{3}}{\left(\omega_{n}-i \mu\right)^{2}+E_{q, c}^{2}(\mathbf{k})+\Delta_{3}^{2}},
\end{aligned}
$$

where $\omega_{n}=(2 n+1) \pi T$ with $n$ being integers. The parameter chemical potential $\mu$ is introduced to calculate the compressibility subsequently. The free energy of the fermions is given by

$$
\begin{aligned}
F_{f}(T, \mu)= & -2 N T \sum_{\omega_{n}} \int \frac{d^{3} \mathbf{k}}{(2 \pi)^{3}} \\
& \times \ln \left[\left(\left(\omega_{n}-i \mu\right)^{2}+E_{q, c}^{\prime 2}(\mathbf{k})\right)^{1 / 2}\right],
\end{aligned}
$$

where

$$
E_{q, c}^{\prime}(\mathbf{k})=\sqrt{E_{q, c}^{2}(\mathbf{k})+\Delta_{3}^{2}}
$$

Carrying out the frequency summation, we obtain

$$
\begin{aligned}
F_{f}(T, \mu)= & -2 N \sum_{\alpha= \pm 1} \int \frac{d^{3} \mathbf{k}}{(2 \pi)^{3}}\left[E_{q, c}^{\prime}(\mathbf{k})\right. \\
& \left.+T \ln \left(1+e^{-\frac{E_{q, c}^{\prime}(\mathbf{k})+\alpha \mu}{T}}\right)\right]
\end{aligned}
$$

which is clearly divergent. In order to get a finite free energy, we redefine $F_{f}(T)-F_{f}(0)$ as $F_{f}(T)$, and get

$$
\begin{aligned}
F_{f}(T, \mu)= & -2 N T \sum_{\alpha= \pm 1} \int \frac{d^{3} \mathbf{k}}{(2 \pi)^{3}} \ln \left(1+e^{-\frac{E_{q, c}^{\prime}(\mathbf{k}) \pm \alpha \mu}{T}}\right) \\
\approx & -2 N T k_{F} \sum_{\alpha= \pm 1} \int \frac{d k_{r} d k_{z}}{(2 \pi)^{2}} \\
& \times \ln \left(1+e^{-\frac{E_{q, c}^{\prime}(\mathbf{k}) \pm \alpha \mu}{T}}\right) .
\end{aligned}
$$

Taking the limit $\mu=0$, we have

$$
F_{f}(T)=-4 N T k_{F} \int \frac{d k_{r} d k_{z}}{(2 \pi)^{2}} \ln \left(1+e^{-\frac{E_{q, c}^{\prime}(\mathbf{k})}{T}}\right)
$$

The specific heat is defined as

$$
C_{v}(T)=-T \frac{\partial^{2} F_{f}(T)}{\partial T^{2}} .
$$

Substituting Eq. (2) into Eqs. (99) and (100), we find that for quadratic NLF system, if $\Delta_{3}=0$, the specific heat reads as

$$
C_{v}(T)=\frac{\pi N k_{F}}{24 A} T ;
$$

If $\Delta_{3}$ is finite, the specific heat satisfies

$$
C_{v}(T) \approx \frac{N k_{F}}{\pi A} \frac{\Delta_{3}^{3}}{T^{2}} e^{-\frac{\Delta_{3}}{T}},
$$

in the limit $T \ll \Delta_{3}$. For cubic NLF system, substituting Eq. (4) into Eqs. (99) and (100), if $\Delta_{3}=0$, we obtain

$$
C_{v}(T)=\frac{20 a_{1} N T^{2 / 3} k_{F}}{9 \pi B^{2 / 3}}
$$

where

$$
a_{1}=\int_{0}^{+\infty} d x x \ln \left(1+e^{-x^{3}}\right) \approx 0.3547
$$

For finite $\Delta_{3}$, we have

$$
C_{v}(T) \approx \frac{N k_{F}}{\pi B^{2 / 3}} \frac{\Delta_{3}^{8 / 3}}{T^{2}} e^{-\frac{\Delta_{3}}{T}}
$$

in the limit $T \ll \Delta_{3}$.

The compressibility is defined as

$$
\kappa(T, \mu)=-\frac{\partial^{2} F_{f}(T, \mu)}{\partial \mu^{2}} .
$$


Substituting Eq. (98) into Eq. (106) and then taking $\mu=0$, we can get the expressions of compressibility for quadratic and cubic NLF systems. Concretely, for quadratic NLF system, the compressibility is given by

$$
\kappa(T)=\frac{N k_{F}}{2 \pi A},
$$

in the case $\Delta_{3}=0$, and

$$
\kappa(T) \approx \frac{N k_{F}}{\pi A} \frac{\Delta_{3}^{2}}{T^{2}} e^{-\frac{\Delta_{3}}{T}},
$$

for finite $\Delta_{3}$ in the limit $T \ll \Delta_{3}$. For cubic NLF system, in the case $\Delta_{3}=0$, the compressibility reads as

$$
\kappa(T)=\frac{2 a_{2} N k_{F}}{\pi B^{2 / 3}} T^{-1 / 3},
$$

where

$$
a_{2}=\int_{0}^{+\infty} d x x \frac{e^{x^{3}}}{\left(1+e^{x^{3}}\right)^{2}} \approx 0.1903 .
$$

As shown in Eq. (109), the compressibility $\kappa$ of cubic NLF system is divergent in the limit $T \rightarrow 0$. This singular behavior of $\kappa$ is closely related to the singular behavior of DOS $\rho(\omega) \sim \omega^{-1 / 3}$, which is divergent in the limit $\omega \rightarrow 0$. Divergence of DOS at the Fermi level indicates that the influence of short-range four-interaction would be remarkable. Indeed, as shown in Sec. $\mathrm{VB}$, in many cases, arbitrarily weak four-fermion interactions could drive the system to become unstable to a new phase. Thus, the singular behavior of $\kappa$ is indeed an indication that the influence of four-fermion interactions is important in cubic NLF system. For finite $\Delta_{3}$,

$$
\kappa(T) \approx \frac{N k_{F}}{\pi B^{2 / 3}} \frac{\Delta_{3}^{2 / 3}}{T} e^{-\frac{\Delta_{3}}{T}},
$$

in the limit $T \ll \Delta_{3}$.

In the RG analysis, the temperature can be introduced through the transformation $T=T_{0} e^{-\ell}$, where $T_{0}$ is the initial value of temperature and $\ell$ is the $R G$ running parameter. For example, this transformation was utilized to calculate the corrections of observable quantities induced by long-range Coulomb interaction in graphene 31, 33], 3D DSM/WSM [34, 35], multi-WSMs [42 45], through incorporating the renormalization of fermion velocities.

We have found that the four-fermion coupling parameters could become divergent at a critical value $\ell_{c}$. It represents that if $T<T_{c}$, where $T_{c}=T_{0} e^{-\ell_{c}}$, the system becomes unstable to a new phase, which may be conventional NLF phase, excitonic insulating phase, or superconducting phase. For the case $\ell<\ell_{c}$, the four-fermion coupling parameters have not flowed to the strong-coupling regime. Thus, if $T>T_{c}$, the system is stable and still in the original phase. Accordingly, the observable quantities take the same forms as the free fermion system, due to that the fermion dispersion is not renormalized by the short-range four-fermion interactions in this case. If $T<T_{c}$, the behaviors of observable quantities are modified obviously, since the system becomes to be in a new phase.

\section{ROLE OF THE GEOMETRY OF THE NODAL LINE}

Thereinbefore, we consider a nodal line with circular shape. It is interesting to verify whether the results are changed by the geometry of the nodal line. In this section, we consider a straight nodal line along the $y$ axis from $-\Lambda_{y}$ to $\Lambda_{y}$. The expressions of Hamiltonian density for quadratic and cubic NLSMs are given by

$$
\mathcal{H}_{0}=A\left[\left(k_{x}^{2}-k_{z}^{2}\right) \sigma_{1}+2 k_{x} k_{z} \sigma_{2}\right]
$$

and

$$
\mathcal{H}_{0}=B\left[\left(k_{x}^{3}-3 k_{x} k_{z}^{2}\right) \sigma_{1}+\left(k_{z}^{3}-3 k_{z} k_{x}^{2}\right) \sigma_{2}\right],
$$

respectively. After tedious calculation and derivation, we find that the expressions of the RG equations for the four-fermion coupling parameters are not changed. For quadratic NLF system, the RG equations are still given by Eqs. (62)-65). The transformations

$$
\frac{\Lambda_{y}}{2 \pi^{2} A} \lambda_{i} \rightarrow \lambda_{i}
$$

with $i=0,1,2,3$, have been used in the derivation. For cubic NLF system, the RG equations are still given by Eqs. (66)- (69). The transformations

$$
\frac{\Lambda_{y}}{2 \pi^{2} B \Lambda} \lambda_{i} \rightarrow \lambda_{i}
$$

with $i=0,1,2,3$, have been utilized in the derivation. Thus, the results shown in former sections are still valid for the system with straight nodal line. We believe that the results also hold on for a system in which the nodal lines take other shapes.

\section{SUMMARY AND DISCUSSION}

In summary, we study the influence of four-fermion interactions on the quadratic and cubic NLF systems. Through RG analysis, we find that arbitrarily weak fourfermion interactions could drive the system to NLSM, excitonic insulator, or superconducting phase, which is determined by the concrete initial conditions and value of fermion flavor. The remarkable interaction effects in quadratic and cubic NLF systems are closely related to the dispersion of fermion excitations.

$\mathrm{Yu}$ et al. predicted that quadratic NLF system may be realized in the candidate materials including $\mathrm{ZrPtGa}$, $\mathrm{V}_{12} \mathrm{P}_{7}, \mathrm{ZrRuAs}$, and cubic NLF system may be realized in $\mathrm{CaAgBi}$ 71]. We expect our theoretical predictions may be verified experimentally in these candidate materials for quadratic and cubic NLF systems in future.

Recently, Volkov and Moroz found that nodal surface fermion system is another strong correlated system in three dimension, since it would be driven to excitonic insulating phase under arbitrarily weak Coulomb interaction [79]. 


\section{ACKNOWLEDGEMENTS}

J.R.W. is grateful to Prof. G.-Z. Liu for the valuable discussions. We acknowledge the support from the National Key R\&D Program of China under Grants 2017YFA0403600 and 2016YFA0300404, the National Natural Science Foundation of China under Grants 11504379, 11674327, 11974356, and U1832209, and the Collaborative Innovation Program of Hefei Science Center CAS under Grant 2019HSC-CIP002. A portion of this work was supported by the High Magnetic Field Laboratory of Anhui Province.

\section{Appendix A: From lattice model to low-energy effective model}

In this section, we show the lattice models for quadratic and cubic NLF systems, and derive the corresponding low-energy effective models.

\section{Quadratic NLF system}

We consider a lattice Hamiltonian for quadratic NLF system as following

$$
\begin{aligned}
\mathcal{H}^{q}= & -t_{q}\left[\cos \left(k_{r}\right)-\cos \left(\frac{k_{r}}{2}\right) \cos \left(\frac{\sqrt{3} k_{z}}{2}\right)\right] \sigma_{1} \\
& +\sqrt{3} t_{q} \sin \left(\frac{k_{r}}{2}\right) \sin \left(\frac{\sqrt{3} k_{z}}{2}\right) \sigma_{2}
\end{aligned}
$$

Expanding it around the nodal line determined by $k_{r}=$ $0, k_{z}=0$, we get

$$
\begin{aligned}
\mathcal{H}^{q} \approx & -t_{q}\left[\left(1-\frac{k_{r}^{2}}{2}\right)-\left(1-\frac{k_{r}^{2}}{8}\right)\left(1-\frac{3 k_{z}^{2}}{8}\right)\right] \sigma_{1} \\
& +\sqrt{3} t_{q} \frac{k_{r}}{2} \frac{\sqrt{3} k_{z}}{2} \sigma_{2} \\
\approx & \frac{3}{8} t_{q}\left[\left(k_{r}^{2}-k_{z}^{2}\right) \sigma_{1}+2 k_{r} k_{z} \sigma_{2}\right] \\
= & A\left[\left(k_{r}^{2}-k_{z}^{2}\right) \sigma_{1}+2 k_{r} k_{z} \sigma_{2}\right]
\end{aligned}
$$

where

$$
A=\frac{3}{8} t_{q}
$$

\section{Cubic NLF system}

We consider a lattice Hamiltonian for cubic NLF system as following

$\mathcal{H}^{c}=t_{c} \sin \left(\frac{\sqrt{3} k_{r}}{2}\right)\left[\cos \left(\frac{3 k_{z}}{2}\right)-\cos \left(\frac{\sqrt{3} k_{r}}{2}\right)\right] \sigma_{1}$

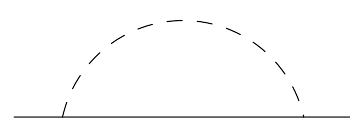

(a)

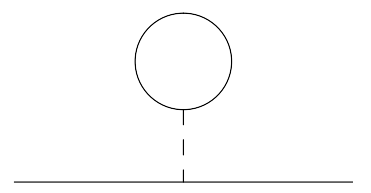

(b)
FIG. 9: Feynman diagrams for the self-energies of fermions induced by four-fermion interactions. Solid line represents the fermion propagator, and dashed line stands for the fourfermion interaction.

$$
-3 \sqrt{3} t_{c} \sin \left(\frac{k_{z}}{2}\right)\left[\cos \left(\frac{k_{z}}{2}\right)-\cos \left(\frac{\sqrt{3} k_{r}}{2}\right)\right] \sigma_{2}
$$

Expanding it around the nodal line decided by $k_{r}=$ $0, k_{z}=0$, we obtain

$$
\begin{aligned}
\mathcal{H}^{c} \approx & t_{c} \frac{\sqrt{3} k_{r}}{2}\left[\left(1-\frac{9 k_{z}^{2}}{8}\right)-\left(1-\frac{3 k_{r}^{2}}{8}\right)\right] \sigma_{1} \\
& -3 \sqrt{3} t_{c} \frac{k_{z}}{2}\left[\left(1-\frac{k_{z}^{2}}{8}\right)-\left(1-\frac{3 k_{r}^{2}}{8}\right)\right] \sigma_{2} \\
= & \frac{3 \sqrt{3} t_{c}}{16}\left[\left(k_{r}^{3}-3 k_{r} k_{z}^{2}\right) \sigma_{1}+\left(k_{z}^{3}-3 k_{z} k_{r}^{2}\right) \sigma_{2}\right] \\
= & B\left[\left(k_{r}^{3}-3 k_{r} k_{z}^{2}\right) \sigma_{1}+\left(k_{z}^{3}-3 k_{z} k_{r}^{2}\right) \sigma_{2}\right],
\end{aligned}
$$

where

$$
B=\frac{3 \sqrt{3} t_{c}}{16}
$$

\section{Appendix B: Fermion propagator}

The fermion propagator for quadratic NLF system takes the form

$$
G_{q 0}(\omega, \mathbf{k})=\frac{1}{-i \omega+\mathcal{H}_{0}^{q}(\mathbf{k})}
$$

where $\mathcal{H}_{0}^{q}$ is given by Eq. (11). The fermion propagator for cubic NLF system can be written as

$$
G_{c 0}(\omega, \mathbf{k})=\frac{1}{-i \omega+\mathcal{H}_{0}^{c}(\mathbf{k})}
$$

where $\mathcal{H}_{0}^{c}$ is expressed by Eq. (3).

\section{Appendix C: Self-energy of the fermions}

The self-energy of fermions induced by Fig. 9(a) is defined as

$$
\Sigma_{a}=\sum_{i=0}^{3} \frac{\lambda_{i}}{N} \int \frac{d \omega}{2 \pi} \int^{\prime} \frac{d^{3} \mathbf{k}}{(2 \pi)^{3}} \sigma_{i} G_{q, c 0}(\omega, \mathbf{k}) \sigma_{i}
$$




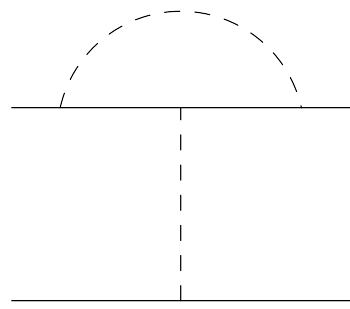

(a)

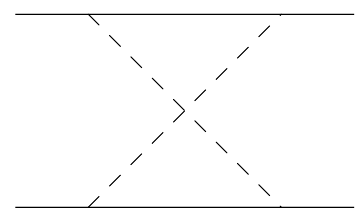

(c)

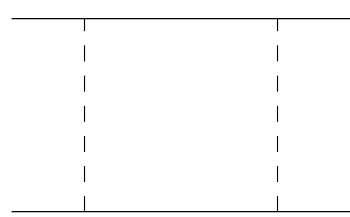

(b)

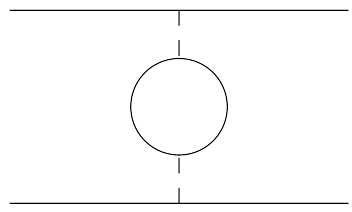

(d)
FIG. 10: One-loop Feynman diagrams for the corrections to the four-fermion couplings. where $\int^{\prime}$ represents that a momentum shell will be properly taken. Figure 9(b) results in the self-energy of fermions as following

$$
\Sigma_{b}=N \sum_{i=0}^{3} \frac{\lambda_{i}}{N} \int \frac{d \omega}{2 \pi} \int^{\prime} \frac{d^{3} \mathbf{k}}{(2 \pi)^{3}} \operatorname{Tr}\left[G_{q, c 0}(\omega, \mathbf{k}) \sigma_{i}\right]
$$

Substituting Eq. (B1) or Eq. (B2) into Eqs. (C1) and (C2), we obtain

$$
\begin{aligned}
& \Sigma_{a}=0, \\
& \Sigma_{b}=0,
\end{aligned}
$$

for both of quadratic and cubic NLF systems. Thus the fermion propagator is not renormalized by the fourfermion interactions to one-loop order.

\section{Appendix D: One-loop order corrections for the four-fermion couplings}

\section{General expressions for the one-loop order corrections}

The correction contributed by Fig. 10(a) is given by

$$
W^{(1)}=\sum_{i=0}^{3} W_{i}^{(1)}
$$

where

$$
W_{i}^{(1)}=\sum_{j=0}^{3} \frac{4 \lambda_{i} \lambda_{j}}{N}\left(\psi^{\dagger} \sigma_{i} \psi\right) \int \frac{d \omega}{2 \pi} \int^{\prime} \frac{d^{3} \mathbf{k}}{(2 \pi)^{3}}\left[\psi^{\dagger} \sigma_{j} G_{q, c 0}(\omega, \mathbf{k}) \sigma_{i} G_{q, c 0}(\omega, \mathbf{k}) \sigma_{j} \psi\right] .
$$

The diagrams as shown in Figs. 10(b) and 10(c) lead to the correction for the four-fermion couplings as following

$$
W^{(2)+(3)}=\sum_{i=0}^{3} \sum_{i \leq j \leq 3} W_{i j}^{(2)+(3)}
$$

where

$$
W_{i j}^{(2)+(3)}=\frac{4 \lambda_{i} \lambda_{j}}{N} \int \frac{d \omega}{2 \pi} \int^{\prime} \frac{d^{3} \mathbf{k}}{(2 \pi)^{3}}\left(\psi^{\dagger} \sigma_{i} G_{q, c 0}(\omega, \mathbf{k}) \sigma_{j} \psi\right)\left\{\psi^{\dagger}\left[\sigma_{j} G_{q, c 0}(\omega, \mathbf{k}) \sigma_{i}+\sigma_{i} G_{q, c 0}(-\omega,-\mathbf{k}) \sigma_{j}\right] \psi\right\} .
$$

The correction for the four-fermion couplings resulting from Fig. 10(d) can be written as

$$
W^{(4)}=\sum_{i=0}^{3} W_{i}^{(4)}
$$

where

$$
W_{i}^{(4)}=-2 \lambda_{i}^{2}\left(\psi^{\dagger} \sigma_{i} \psi\right)\left(\psi^{\dagger} \sigma_{i} \psi\right) \int \frac{d \omega}{2 \pi} \int^{\prime} \frac{d^{3} \mathbf{k}}{(2 \pi)^{3}} \operatorname{Tr}\left[\sigma_{i} G_{q, c 0}(\omega, \mathbf{k}) \sigma_{i} G_{q, c 0}(\omega, \mathbf{k})\right] .
$$

A momentum shell $b \Lambda<\sqrt{k_{r}^{2}+k_{z}^{2}}<\Lambda$ with $b=e^{-\ell}$ will be utilized in the derivation, where $\ell$ stands for the RG running parameter. 


\section{Results for quadratic NLF}

Substituting Eq. (B1) into Eqs. (D1)- (D6), we obtain

$$
\begin{aligned}
W^{(1)}= & \lambda_{1}\left(\lambda_{0}-\lambda_{1}+\lambda_{2}+\lambda_{3}\right) \frac{1}{N} \frac{k_{F}}{2 \pi A} \ell\left(\psi^{\dagger} \sigma_{1} \psi\right)^{2}+\lambda_{2}\left(\lambda_{0}+\lambda_{1}-\lambda_{2}+\lambda_{3}\right) \frac{1}{N} \frac{k_{F}}{2 \pi A} \ell\left(\psi^{\dagger} \sigma_{2} \psi\right)^{2} \\
& +\lambda_{3}\left(\lambda_{0}+\lambda_{1}+\lambda_{2}-\lambda_{3}\right) \frac{1}{N} \frac{k_{F}}{\pi A} \ell\left(\psi^{\dagger} \sigma_{3} \psi\right)^{2}, \\
W^{(2)+(3)=} & \left(\lambda_{0} \lambda_{1}+\lambda_{0} \lambda_{2}\right) \frac{1}{N} \frac{k_{F}}{2 \pi A} \ell\left(\psi^{\dagger} \sigma_{0} \psi\right)^{2}+\left(\sum_{i=0}^{3} \lambda_{i}^{2}+\lambda_{1} \lambda_{2}-2 \lambda_{2} \lambda_{3}\right) \frac{1}{N} \frac{k_{F}}{2 \pi A} \ell\left(\psi^{\dagger} \sigma_{1} \psi\right)^{2} \\
& +\left(\sum_{i=0}^{3} \lambda_{i}^{2}+\lambda_{1} \lambda_{2}-2 \lambda_{1} \lambda_{3}\right) \frac{1}{N} \frac{k_{F}}{2 \pi A} \ell\left(\psi^{\dagger} \sigma_{2} \psi\right)^{2}+\left(-2 \lambda_{1} \lambda_{2}+\lambda_{1} \lambda_{3}+\lambda_{2} \lambda_{3}\right) \frac{1}{N} \frac{k_{F}}{2 \pi A} \ell\left(\psi^{\dagger} \sigma_{3} \psi\right)^{2},(1) \\
W^{(4)}= & \lambda_{1}^{2} \frac{k_{F}}{2 \pi A} \ell\left(\psi^{\dagger} \sigma_{1} \psi\right)^{2}+\lambda_{2}^{2} \frac{k_{F}}{2 \pi A} \ell\left(\psi^{\dagger} \sigma_{2} \psi\right)^{2}+\lambda_{3}^{2} \frac{k_{F}}{\pi A} \ell\left(\psi^{\dagger} \sigma_{3} \psi\right)^{2} .
\end{aligned}
$$

From Eqs. (D7)-(D9), we get

$$
W=W^{(1)}+W^{(2)+(3)}+W^{(4)}=\sum_{i=0}^{3} \delta \lambda_{i}\left(\psi^{\dagger} \sigma_{i} \psi\right)^{2}
$$

where

$$
\begin{aligned}
\delta \lambda_{0} & =\left(\lambda_{0} \lambda_{1}+\lambda_{0} \lambda_{2}\right) \frac{1}{N} \frac{k_{F}}{2 \pi A} \ell \\
\delta \lambda_{1} & =\left[\left(\lambda_{0}^{2}+\lambda_{2}^{2}+\lambda_{3}^{2}+\lambda_{0} \lambda_{1}+2 \lambda_{1} \lambda_{2}+\lambda_{1} \lambda_{3}-2 \lambda_{2} \lambda_{3}\right) \frac{1}{N}+\lambda_{1}^{2}\right] \frac{k_{F}}{2 \pi A} \ell \\
\delta \lambda_{2} & =\left[\left(\lambda_{0}^{2}+\lambda_{1}^{2}+\lambda_{3}^{2}+\lambda_{0} \lambda_{2}+2 \lambda_{1} \lambda_{2}-2 \lambda_{1} \lambda_{3}+\lambda_{2} \lambda_{3}\right) \frac{1}{N}+\lambda_{2}^{2}\right] \frac{k_{F}}{2 \pi A} \ell \\
\delta \lambda_{3} & =\left[\left(-2 \lambda_{3}^{2}+2 \lambda_{0} \lambda_{3}-2 \lambda_{1} \lambda_{2}+3 \lambda_{1} \lambda_{3}+3 \lambda_{2} \lambda_{3}\right) \frac{1}{N}+2 \lambda_{3}^{2}\right] \frac{k_{F}}{2 \pi A} \ell
\end{aligned}
$$

\section{Results for cubic NLF}

Substituting Eq. (B2) into Eqs. (D1)- (D6), we get

$$
\begin{aligned}
W^{(1)}= & \lambda_{1}\left(\lambda_{0}-\lambda_{1}+\lambda_{2}+\lambda_{3}\right) \frac{1}{N} \frac{k_{F}}{2 \pi B \Lambda} \ell\left(\psi^{\dagger} \sigma_{1} \psi\right)^{2}+\lambda_{2}\left(\lambda_{0}+\lambda_{1}-\lambda_{2}+\lambda_{3}\right) \frac{1}{N} \frac{k_{F}}{2 \pi B \Lambda} \ell\left(\psi^{\dagger} \sigma_{2} \psi\right)^{2} \\
& +\lambda_{3}\left(\lambda_{0}+\lambda_{1}+\lambda_{2}-\lambda_{3}\right) \frac{1}{N} \frac{k_{F}}{\pi B \Lambda} \ell\left(\psi^{\dagger} \sigma_{3} \psi\right)^{2}, \\
W^{(2)+(3)}= & \left(\lambda_{1} \lambda_{3}+\lambda_{2} \lambda_{3}\right) \frac{1}{N} \frac{k_{F}}{2 \pi B \Lambda} \ell\left(\psi^{\dagger} \sigma_{0} \psi\right)^{2}+\left(\lambda_{0} \lambda_{3}-2 \lambda_{2} \lambda_{3}\right) \frac{1}{N} \frac{k_{F}}{2 \pi B \Lambda} \ell\left(\psi^{\dagger} \sigma_{1} \psi\right)^{2} \\
& +\left(\lambda_{0} \lambda_{3}-2 \lambda_{1} \lambda_{3}\right) \frac{1}{N} \frac{k_{F}}{2 \pi B \Lambda} \ell\left(\psi^{\dagger} \sigma_{2} \psi\right)^{2}+\left(\lambda_{0} \lambda_{1}+\lambda_{0} \lambda_{2}-2 \lambda_{1} \lambda_{2}\right) \frac{1}{N} \frac{k_{F}}{2 \pi B \Lambda} \ell\left(\psi^{\dagger} \sigma_{3} \psi\right)^{2}, \\
W^{(4)}= & \lambda_{1}^{2} \frac{k_{F}}{2 \pi B \Lambda} \ell\left(\psi^{\dagger} \sigma_{1} \psi\right)^{2}+\lambda_{2}^{2} \frac{k_{F}}{2 \pi B \Lambda} \ell\left(\psi^{\dagger} \sigma_{2} \psi\right)^{2}+\lambda_{3}^{2} \frac{k_{F}}{\pi B \Lambda} \ell\left(\psi^{\dagger} \sigma_{3} \psi\right)^{2} .
\end{aligned}
$$

From Eqs. (D15)-(D17), we find

$$
W=W^{(1)}+W^{(2)+(3)}+W^{(4)}=\sum_{i=0}^{3} \delta \lambda_{i}\left(\psi^{\dagger} \sigma_{i} \psi\right)^{2},
$$


where

$$
\begin{aligned}
\delta \lambda_{0} & =\left(\lambda_{1} \lambda_{3}+\lambda_{2} \lambda_{3}\right) \frac{1}{N} \frac{k_{F}}{2 \pi B \Lambda} \ell, \\
\delta \lambda_{1} & =\left[\left(-\lambda_{1}^{2}+\lambda_{0} \lambda_{1}+\lambda_{0} \lambda_{3}+\lambda_{1} \lambda_{2}+\lambda_{1} \lambda_{3}-2 \lambda_{2} \lambda_{3}\right) \frac{1}{N}+\lambda_{1}^{2}\right] \frac{k_{F}}{2 \pi B \Lambda} \ell, \\
\delta \lambda_{2} & =\left[\left(-\lambda_{2}^{2}+\lambda_{0} \lambda_{2}+\lambda_{0} \lambda_{3}+\lambda_{1} \lambda_{2}-2 \lambda_{1} \lambda_{3}+\lambda_{2} \lambda_{3}\right) \frac{1}{N}+\lambda_{2}^{2}\right] \frac{k_{F}}{2 \pi B \Lambda} \ell, \\
\delta \lambda_{3} & =\left[\left(-2 \lambda_{3}^{2}+\lambda_{0} \lambda_{1}+\lambda_{0} \lambda_{2}+2 \lambda_{0} \lambda_{3}-2 \lambda_{1} \lambda_{2}+2 \lambda_{1} \lambda_{3}+2 \lambda_{2} \lambda_{3}\right) \frac{1}{N}+2 \lambda_{3}^{2}\right] \frac{k_{F}}{2 \pi B \Lambda} \ell .
\end{aligned}
$$

\section{Appendix E: Derivation of the RG equations}

\section{Quadratic NLF}

The action for quadratic NLFs is

$$
S_{\psi}=\int \frac{d \omega}{2 \pi} \frac{d^{3} \mathbf{k}}{(2 \pi)^{3}} \psi^{\dagger}(\omega, \mathbf{k})\left[-i \omega+A\left(k_{r}^{2}-k_{z}^{2}\right) \sigma_{1}+2 A k_{r} k_{z} \sigma_{2}\right] \psi(\omega, \mathbf{k}),
$$

where $k_{r} \equiv k_{\perp}-k_{F} \equiv \sqrt{k_{x}^{2}+k_{y}^{2}}-k_{F}$. In the low-energy regime, the action for quadratic NLF can be also written as

$$
S_{\psi} \approx k_{F} \int \frac{d \omega}{2 \pi} \frac{d k_{r}}{2 \pi} \frac{d k_{z}}{2 \pi} \psi^{\dagger}(\omega, \mathbf{k})\left[-i \omega+A\left(k_{r}^{2}-k_{z}^{2}\right) \sigma_{1}+2 A k_{r} k_{z} \sigma_{2}\right] \psi(\omega, \mathbf{k})
$$

Employing the transformations

$$
\begin{aligned}
k_{r} & =k_{r}^{\prime} e^{-\frac{\ell}{2}}, \\
k_{z} & =k_{z}^{\prime} e^{-\frac{\ell}{2}}, \\
\omega & =\omega^{\prime} e^{-\ell} \\
\psi & =\psi^{\prime} e^{\frac{3}{2} \ell} \\
A & =A^{\prime},
\end{aligned}
$$

the action of the quadratic NLFs becomes

$$
S_{\psi^{\prime}}=k_{F} \int \frac{d \omega^{\prime}}{2 \pi} \frac{d k_{r}^{\prime}}{2 \pi} \frac{d k_{z}^{\prime}}{2 \pi} \psi^{\prime \dagger}\left(\omega^{\prime}, \mathbf{k}^{\prime}\right)\left[-i \omega^{\prime}+A^{\prime}\left(k_{r}^{\prime 2}-k_{z}^{\prime 2}\right) \sigma_{1}+2 A^{\prime} k_{r}^{\prime} k_{z}^{\prime} \sigma_{2}\right] \psi^{\prime}\left(\omega^{\prime}, \mathbf{k}^{\prime}\right),
$$

which recovers the form of the original action.

The action for the four-fermion interactions between quadratic NLFs is given by

$$
\begin{aligned}
S_{\psi^{4}}= & \frac{1}{N} \sum_{i=0}^{3} \lambda_{i} \int \frac{d \omega_{1}}{2 \pi} \frac{d^{3} \mathbf{k}_{1}}{(2 \pi)^{3}} \frac{d \omega_{2}}{2 \pi} \frac{d^{3} \mathbf{k}_{2}}{(2 \pi)^{3}} \frac{d \omega_{3}}{2 \pi} \frac{d^{3} \mathbf{k}_{3}}{(2 \pi)^{3}} \psi^{\dagger}\left(\omega_{1}, \mathbf{k}_{1}\right) \sigma_{i} \psi\left(\omega_{2}, \mathbf{k}_{2}\right) \psi^{\dagger}\left(\omega_{3}, \mathbf{k}_{3}\right) \sigma_{i} \psi\left(\omega_{1}-\omega_{2}+\omega_{3}, \mathbf{k}_{1}-\mathbf{k}_{2}+\mathbf{k}_{3}\right) \\
\approx & \frac{1}{N} \sum_{i=0}^{3} \lambda_{i} k_{F}^{3} \int \frac{d \omega_{1}}{2 \pi} \frac{d k_{1 r}}{2 \pi} \frac{d k_{1 z}}{2 \pi} \frac{d \omega_{2}}{2 \pi} \frac{d k_{2 r}}{2 \pi} \frac{d k_{2 z}}{2 \pi} \frac{d \omega_{3}}{2 \pi} \frac{d k_{3 r}}{2 \pi} \frac{d k_{3 z}}{2 \pi} \psi^{\dagger}\left(\omega_{1}, \mathbf{k}_{1}\right) \sigma_{i} \psi\left(\omega_{2}, \mathbf{k}_{2}\right) \psi^{\dagger}\left(\omega_{3}, \mathbf{k}_{3}\right) \sigma_{i} \\
& \times \psi\left(\omega_{1}-\omega_{2}+\omega_{3}, \mathbf{k}_{1}-\mathbf{k}_{2}+\mathbf{k}_{3}\right) .
\end{aligned}
$$

Incorporating the one-loop order corrections, the action becomes

$$
\begin{aligned}
S_{\psi^{4}}= & \frac{1}{N} \sum_{i=0}^{3}\left(\lambda_{i}+\delta \lambda_{i}\right) k_{F}^{3} \int \frac{d \omega_{1}}{2 \pi} \frac{d k_{1 r}}{2 \pi} \frac{d k_{1 z}}{2 \pi} \frac{d \omega_{2}}{2 \pi} \frac{d k_{2 r}}{2 \pi} \frac{d k_{2 z}}{2 \pi} \frac{d \omega_{3}}{2 \pi} \frac{d k_{3 r}}{2 \pi} \frac{d k_{3 z}}{2 \pi} \psi^{\dagger}\left(\omega_{1}, \mathbf{k}_{1}\right) \sigma_{i} \psi\left(\omega_{2}, \mathbf{k}_{2}\right) \psi^{\dagger}\left(\omega_{3}, \mathbf{k}_{3}\right) \sigma_{i} \\
& \times \psi\left(\omega_{1}-\omega_{2}+\omega_{3}, \mathbf{k}_{1}-\mathbf{k}_{2}+\mathbf{k}_{3}\right) .
\end{aligned}
$$


Adopting the transformations shown in Eqs. (E3)-(E6), and

$$
\lambda_{i}^{\prime}=\lambda_{i}+\delta \lambda_{i}
$$

the action can be written as

$$
\begin{aligned}
S_{\psi^{\prime 4}}= & \frac{1}{N} \sum_{i=0}^{3} \lambda_{i}^{\prime} \int \frac{d \omega_{1}^{\prime}}{2 \pi} \frac{d k_{1 r}^{\prime}}{2 \pi} \frac{d k_{1 z}^{\prime}}{2 \pi} \frac{d \omega_{2}^{\prime}}{2 \pi} \frac{d k_{2 r}^{\prime}}{2 \pi} \frac{d k_{2 z}^{\prime}}{2 \pi} \frac{d \omega_{3}^{\prime}}{2 \pi} \frac{d k_{3 r}^{\prime}}{2 \pi} \frac{d k_{3 z}^{\prime}}{2 \pi} \psi^{\prime \dagger}\left(\omega_{1}^{\prime}, \mathbf{k}_{1}^{\prime}\right) \sigma_{i} \psi^{\prime}\left(\omega_{2}^{\prime}, \mathbf{k}_{2}^{\prime}\right) \psi^{\prime \dagger}\left(\omega_{3}^{\prime}, \mathbf{k}_{3}^{\prime}\right) \sigma_{i} \\
& \times \psi^{\prime}\left(\omega_{1}^{\prime}-\omega_{2}^{\prime}+\omega_{3}^{\prime}, \mathbf{k}_{1}^{\prime}-\mathbf{k}_{2}^{\prime}+\mathbf{k}_{3}^{\prime}\right)
\end{aligned}
$$

which recovers the original form of the action. From Eq. (E11), we obtain

$$
\frac{d \lambda_{i}}{d \ell}=\frac{d \delta \lambda_{i}}{d \ell}
$$

Substituting Eqs. (D11)-(D14) into Eq. (E13), we get the RG equations

$$
\begin{aligned}
\frac{d \lambda_{0}}{d \ell} & =\left(\lambda_{0} \lambda_{1}+\lambda_{0} \lambda_{2}\right) \frac{1}{N} \\
\frac{d \lambda_{1}}{d \ell} & =\left(\lambda_{0}^{2}+\lambda_{2}^{2}+\lambda_{3}^{2}+\lambda_{0} \lambda_{1}+2 \lambda_{1} \lambda_{2}+\lambda_{1} \lambda_{3}-2 \lambda_{2} \lambda_{3}\right) \frac{1}{N}+\lambda_{1}^{2} \\
\frac{d \lambda_{2}}{d \ell} & =\left(\lambda_{0}^{2}+\lambda_{1}^{2}+\lambda_{3}^{2}+\lambda_{0} \lambda_{2}+2 \lambda_{1} \lambda_{2}-2 \lambda_{1} \lambda_{3}+\lambda_{2} \lambda_{3}\right) \frac{1}{N}+\lambda_{2}^{2}, \\
\frac{d \lambda_{3}}{d \ell} & =\left(-2 \lambda_{3}^{2}+2 \lambda_{0} \lambda_{3}-2 \lambda_{1} \lambda_{2}+3 \lambda_{1} \lambda_{3}+3 \lambda_{2} \lambda_{3}\right) \frac{1}{N}+2 \lambda_{3}^{2} .
\end{aligned}
$$

The transformations

$$
\frac{k_{F}}{2 \pi A} \lambda_{i} \rightarrow \lambda_{i}
$$

with $i=0,1,2,3$ have been used.

\section{Cubic NLF}

The action for cubic NLFs takes the form

$$
S_{\psi}=\int \frac{d \omega}{2 \pi} \frac{d^{3} \mathbf{k}}{(2 \pi)^{3}} \psi^{\dagger}(\omega, \mathbf{k})\left[-i \omega+B\left(k_{r}^{3}-3 k_{r} k_{z}^{2}\right) \sigma_{1}+B\left(k_{z}^{3}-3 k_{r} k_{z}^{2}\right) \sigma_{2}\right] \psi(\omega, \mathbf{k}),
$$

which is equivalent to

$$
S_{\psi} \approx k_{F} \int \frac{d \omega}{2 \pi} \frac{d k_{r}}{2 \pi} \frac{d k_{z}}{2 \pi} \psi^{\dagger}(\omega, \mathbf{k})\left[-i \omega+B\left(k_{r}^{3}-3 k_{r} k_{z}^{2}\right) \sigma_{1}+B\left(k_{z}^{3}-3 k_{r} k_{z}^{2}\right) \sigma_{2}\right] \psi(\omega, \mathbf{k}) .
$$

Using the transformations

$$
\begin{aligned}
k_{r} & =k_{r}^{\prime} e^{-\frac{\ell}{3}}, \\
k_{z} & =k_{z}^{\prime} e^{-\frac{\ell}{3}}, \\
\omega & =\omega^{\prime} e^{-\ell}, \\
\psi & =\psi^{\prime} e^{\frac{4}{3} \ell}, \\
B & =B^{\prime},
\end{aligned}
$$

the action can be written as

$$
S_{\psi^{\prime}}=k_{F} \int \frac{d \omega^{\prime}}{2 \pi} \frac{d k_{r}^{\prime}}{2 \pi} \frac{d k_{z}^{\prime}}{2 \pi} \psi^{\prime \dagger}\left(\omega^{\prime}, \mathbf{k}^{\prime}\right)\left[-i \omega^{\prime}+B^{\prime}\left(k_{r}^{\prime 3}-3 k_{r}^{\prime} k_{z}^{\prime 2}\right) \sigma_{1}+B^{\prime}\left(k_{z}^{\prime 3}-3 k_{r}^{\prime} k_{z}^{\prime 2}\right) \sigma_{2}\right] \psi^{\prime}\left(\omega^{\prime}, \mathbf{k}^{\prime}\right),
$$

which has the same form as the original action. 
The action describing the four-fermion interactions between cubic NLFs is given by

$$
\begin{aligned}
S_{\psi^{4}}= & \frac{1}{N} \sum_{i=0}^{3} \lambda_{i} k_{F}^{3} \int \frac{d \omega_{1}}{2 \pi} \frac{d k_{1 r}}{2 \pi} \frac{d k_{1 z}}{2 \pi} \frac{d \omega_{2}}{2 \pi} \frac{d k_{2 r}}{2 \pi} \frac{d k_{2 z}}{2 \pi} \frac{d \omega_{3}}{2 \pi} \frac{d k_{3 r}}{2 \pi} \frac{d k_{3 z}}{2 \pi} \psi^{\dagger}\left(\omega_{1}, \mathbf{k}_{1}\right) \sigma_{i} \psi\left(\omega_{2}, \mathbf{k}_{2}\right) \psi^{\dagger}\left(\omega_{3}, \mathbf{k}_{3}\right) \sigma_{i} \\
& \times \psi\left(\omega_{1}-\omega_{2}+\omega_{3}, \mathbf{k}_{1}-\mathbf{k}_{2}+\mathbf{k}_{3}\right) .
\end{aligned}
$$

Including one-loop order corrections, the action becomes

$$
\begin{aligned}
S_{\psi^{4}}= & \frac{1}{N} \sum_{i=0}^{3}\left(\lambda_{i}+\delta \lambda_{i}\right) k_{F}^{3} \int \frac{d \omega_{1}}{2 \pi} \frac{d k_{1 r}}{2 \pi} \frac{d k_{1 z}}{2 \pi} \frac{d \omega_{2}}{2 \pi} \frac{d k_{2 r}}{2 \pi} \frac{d k_{2 z}}{2 \pi} \frac{d \omega_{3}}{2 \pi} \frac{d k_{3 r}}{2 \pi} \frac{d k_{3 z}}{2 \pi} \psi^{\dagger}\left(\omega_{1}, \mathbf{k}_{1}\right) \sigma_{i} \psi\left(\omega_{2}, \mathbf{k}_{2}\right) \psi^{\dagger}\left(\omega_{3}, \mathbf{k}_{3}\right) \sigma_{i} \\
& \times \psi\left(\omega_{1}-\omega_{2}+\omega_{3}, \mathbf{k}_{1}-\mathbf{k}_{2}+\mathbf{k}_{3}\right) .
\end{aligned}
$$

Utilizing the transformations shown in Eqs. (E21)-(E24), and

$$
\lambda_{i}^{\prime}=\left(\lambda_{i}+\delta \lambda_{i}\right) e^{\frac{1}{3} \ell} \approx \lambda_{i}+\lambda_{i} \frac{1}{3} \ell+\delta \lambda_{i},
$$

the action becomes

$$
\begin{aligned}
S_{\psi^{\prime 4}}= & \sum_{i=0}^{3} \lambda_{i}^{\prime} k_{F}^{3} \int \frac{d \omega_{1}^{\prime}}{2 \pi} \frac{d k_{1 r}^{\prime}}{2 \pi} \frac{d k_{1 z}^{\prime}}{2 \pi} \frac{d \omega_{2}^{\prime}}{2 \pi} \frac{d k_{2 r}^{\prime}}{2 \pi} \frac{d k_{2 z}^{\prime}}{2 \pi} \frac{d \omega_{3}^{\prime}}{2 \pi} \frac{d k_{3 r}^{\prime}}{2 \pi} \frac{d k_{3 z}^{\prime}}{2 \pi} \psi^{\prime \dagger}\left(\omega_{1}^{\prime}, \mathbf{k}_{1}^{\prime}\right) \sigma_{i} \psi^{\prime}\left(\omega_{2}^{\prime}, \mathbf{k}_{2}^{\prime}\right) \psi^{\prime \dagger}\left(\omega_{3}^{\prime}, \mathbf{k}_{3}^{\prime}\right) \sigma_{i} \\
& \times \psi^{\prime}\left(\omega_{1}^{\prime}-\omega_{2}^{\prime}+\omega_{3}^{\prime}, \mathbf{k}_{1}^{\prime}-\mathbf{k}_{2}^{\prime}+\mathbf{k}_{3}^{\prime}\right),
\end{aligned}
$$

which has the same form of the original action. According to Eq. (E29), the RG equation for $\lambda_{i}$ is given by

$$
\frac{d \lambda_{i}}{d \ell}=\frac{1}{3} \lambda_{i}+\frac{d \delta \lambda_{i}}{d \ell}
$$

Substituting Eqs. (D19)-(D22) into Eq. (E31), the RG equations for $\lambda_{i}$ can be written as

$$
\begin{aligned}
\frac{d \lambda_{0}}{d \ell} & =\frac{1}{3} \lambda_{0}+\left(\lambda_{1} \lambda_{3}+\lambda_{2} \lambda_{3}\right) \frac{1}{N}, \\
\frac{d \lambda_{1}}{d \ell} & =\frac{1}{3} \lambda_{1}+\left(-\lambda_{1}^{2}+\lambda_{0} \lambda_{1}+\lambda_{0} \lambda_{3}+\lambda_{1} \lambda_{2}+\lambda_{1} \lambda_{3}-2 \lambda_{2} \lambda_{3}\right) \frac{1}{N}+\lambda_{1}^{2}, \\
\frac{d \lambda_{2}}{d \ell} & =\frac{1}{3} \lambda_{2}+\left(-\lambda_{2}^{2}+\lambda_{0} \lambda_{2}+\lambda_{0} \lambda_{3}+\lambda_{1} \lambda_{2}-2 \lambda_{1} \lambda_{3}+\lambda_{2} \lambda_{3}\right) \frac{1}{N}+\lambda_{2}^{2}, \\
\frac{d \lambda_{3}}{d \ell} & =\frac{1}{3} \lambda_{3}+\left(-2 \lambda_{3}^{2}+\lambda_{0} \lambda_{1}+\lambda_{0} \lambda_{2}+2 \lambda_{0} \lambda_{3}-2 \lambda_{1} \lambda_{2}+2 \lambda_{1} \lambda_{3}+2 \lambda_{2} \lambda_{3}\right) \frac{1}{N}+2 \lambda_{3}^{2} .
\end{aligned}
$$

The transformations

$$
\frac{k_{F}}{2 \pi B \Lambda} \lambda_{i} \rightarrow \lambda_{i}
$$

with $i=0,1,2,3$ have been adopted.

[1] V. N. Kotov, B. Uchoa, V. M. Pereira, F. Guinea, and A. H. Castro Neto, Electron-electron interactions in graphene: Current status and perspectives, Rev. Mod. Phys. 84, 1067 (2012).

[2] O. Vafek and A. Vishwanath, Dirac fermions in solids: From high- $\mathrm{T}_{c}$ cuprates and graphene to topological insulators and Weyl semimetals, Annu. Rev. Condens. Matter
Phys. 5, 83 (2014).

[3] T. O. Wehling, A. M. Black-Schaffer, and A. V. Balatsky, Dirac materials, Adv. Phys. 63, 1 (2014).

[4] X. Wan, A. M. Turner, A. Vishwanath, and S. Y. Savrasov, Topological semimetal and Fermi-arc surface states in the electronic structure of pyrochlore iridates, Phys. Rev. B 83, 205101 (2011). 
[5] H. Weng, X. Dai, and Z. Fang, Topological semimetals predicted from first-principles calculations, J. Phys.: Condens. Matter 28, 303001 (2016).

[6] C. Fang, H. Weng, X. Dai, and Z. Fang, Topological nodal line semimetals, Chin. Phys. B 25, 117106 (2016).

[7] B. Yan and C. Felser, Topological materials: Weyl semimetals, Annu. Rev. Condens. Matter Phys. 8, 337 (2017).

[8] M. Z. Hasan, S.-Y. Xu, I. Belopolski, and S.-M. Huang, Discovery of Weyl fermion semimetals and topological Fermi arc states, Annu. Rev. Condens. Matter Phys. 8, 289 (2017).

[9] N. P. Armitage, E. J. Mele, and A. Vishwanath, Weyl and Dirac semimetals in three-dimensional solids, Rev. Mod. Phys. 90, 015001 (2018).

[10] H. Wang and J. Wang, Electron transport in Dirac and Weyl semimetals, Chin. Phys. B 27, 107402 (2018).

[11] B. Lv, T. Qian, and H. Ding, Angle-resolved photoemission spectroscopy and its application to topological materials, Nat. Rev. Phys. 1, 609 (2019).

[12] J. Kruthoff, J. de Boer, J. van Wezel, C. L. Kane, and R.-J. Slager, Topological classification of crystalline insulators through band structure combinatorics, Phys. Rev. X 7, 041069 (2017).

[13] F. Tang, H. C. Po, A. Vishwanath, and X. Wan, Comprehensive search for topological materials using symmetry indicators, Nature 566, 486 (2019).

[14] T. Zhang, Y. Jiang, Z. Song, H. Huang, Y. He, Z. Fang, H. Weng, and C. Fang, Catalogue of topological electronic materials, Nature 566, 475 (2019).

[15] M. G. Vergniory, L. Elcoro, C. Felser, N. Regnault, B. A. Bernevig, and Z. Wang, A complete catalogue of highquality topological materials, Nature 566, 480 (2019).

[16] B. Skinner and L. Fu, Large, nonsaturating thermopower in a quantizing magnetic field, Sci. Adv. 4, eaat2621 (2018).

[17] M. Markov. S. E. Razaei, S. N. Sadeghi, K. Esfarjani, and M. Zebarjadi, Thermoelectric properties of semimetals, Phys. Rev. Materials 3, 095401 (2019).

[18] M. Neupane, S.-Y. Xu, R. Sankar, N. Alidoust, G. Bian, C. Liu, I. Belopolski, T.-R. Chang, H.-T. Jeng, H. Lin, A. Bansil, F. Chou, and M. Z. Hasan, Observation of a three-dimensional topological Dirac semimetal phase in high-mobility $\mathrm{Cd}_{3} \mathrm{As}_{2}$, Nat. Commun. 5, 3786 (2014).

[19] Z. K. Liu, B. Zhou, Y. Zhang, Z. J. Wang, H. M. Weng, D. Prabhakaran, S.-K. Mo, Z. X. Shen, Z. Fang, X. Dai, Z. Hussain, and Y. L. Chen, Discovery of a threedimensional topological Dirac semimetal, Na $\mathrm{Bi}$, Science 343, 864 (2014).

[20] S.-Y. Xu, I. Belopolski, N. Alidoust, M. Neupane, G. Bian, C. Zhang, R. Sankar, G. Chang, Z. Yuan, C.-C. Lee, S.-M. Huang, H. Zheng, J. Ma, D. S. Sanchez, B. Wang, A. Bansil, F. Chou, P. P. Shibayev, H. Lin, S. Jia, and M. Z. Hasan, Discovery of a Weyl fermion semimetal and topological Fermi arcs, Science 349, 613 (2015).

[21] B. Q. Lv, H. M. Weng, B. B. Fu, X. P. Wang, H. Miao, J. Ma, P. Richard, X. C. Huang, L. X. Zhao, G. F. Chen, Z. Fang, X. Dai, T. Qian, and H. Ding, Experimental discovery of Weyl semimetal TaAs, Phys. Rev. X 5, 031013 (2015).

[22] G. Bian, T.-R. Chang, R. Sankar, S.-Y. Xu, H. Zheng, T. Neupert, C.-K. Chiu, S.-M. Huang, G. Chang, I. Belopolski, D. S. Sanchez, M. Neupane, N. Alidoust, C. Liu, B. Wang, C.-C. Lee, H.-T. Jeng, C. Zhang, Z. Yuan, S.
Jia, A. Bansil, F. Chou, H. Lin, and M. Z. Hasan, Topological nodal-line fermions in spin-orbit metal $\mathrm{PbTaSe}_{2}$, Nat. Commun. 7, 10556 (2016).

[23] M. Neupane, I. Belopolski, M. M. Hosen, D. S. Sanchez, R. Sankar, M. Szlawska, S.-Y. Xu, K. Dimitri, N. Dhakal, P. Maldonado, P. M. Oppeneer, D. Kaczorowski, F. Chou, M. Z. Hasan, and T. Durakiewicz, Observation of topological nodal fermion semimetal phase in $\mathrm{ZrSiS}$, Phys. Rev. B 93, 201104(R) (2016).

[24] L. M. Schoop, M. N. Ali, C. Strasser, A. Topp, A. Varykhalov, D. Marchenko, V. Duppel, S. S. P. Parkin, B. V. Lotsch, and C. R. Ast, Dirac cone protected by non-symmorphic symmetry and three-dimensional Dirac line node in ZrSiS, Nat. Commun. 7, 11696 (2016).

[25] M. M. Hosen, K. Dimitri, I. Belopolski, P. Maldonado, R. Sankar, N. Dhakal, G. Dhakal, T. Cole, P. M. Oppeneer, D. Kaczorowski, F. Chou, M. Z. Hasan, T. Durakiewicz, and M. Neupane, Tunability of the topological nodalline semimetal phase in $\mathrm{ZrSi} X$-type materials $(X=\mathrm{S}$, Se, Te), Phys. Rev. B 95, 161101(R) (2017).

[26] D. Takane, Z. Wang, S. Souma, K. Nakayama, C. X. Trang, T. Sato, T. Takahashi, and Y. Ando, Dirac-node arc in the topological line-node semimetal HfSiS, Phys. Rev. B 94, 121108(R) (2016).

[27] C.-J. Yi, B. Q. Lv, Q. S. Wu. B.-B. Fu, X. Gao, M. Yang, X.-L. Peng, M. Li, Y.-B. Huang, P. Richard, M. Shi, G. Li, O. V. Yazyev, Y.-G. Shi, T. Qian, and H. Ding, Observation of a nodal chain with Dirac surface states in $\mathrm{TiB}_{2}$, Phys. Rev. B 97, 201107(R) (2018).

[28] Z. Liu, R. Luo, P. Guo, Q. Wang, S. Sun, C. Li, S. Thirupathaiah, A. Fedorov, D. Shen, K. Liu, H. Lei, and S. Wang, Experimental observation of Dirac nodal link in centrosymmetric semimetal $\mathrm{TiB}_{2}$, Phys. Rev. X 8, 031044 (2018).

[29] G. F. Giuliani and G. Vignale, Quantum Theory of the Electron Liquid (Cambridge University Press, 2005).

[30] J. González, F. Guinea, and M. A. H. Vozmediano, Marginal-Fermi-liquid behavior from two-dimensional Coulomb interaction, Phys. Rev. B 59, R2474(R) (1999).

[31] D. E. Sheehy and J. Schmalian, Quantum critical scaling in graphene, Phys. Rev. Lett. 99, 226803 (2007).

[32] J. Hofmann, E. Barnes, and S. Das Sarma, Why does graphene behave as a weakly interacting system?, Phys. Rev. Lett. 113, 105502 (2014).

[33] J.-R. Wang and G.-Z. Liu, Influence of Coulomb interaction on the anisotropic Dirac cone in graphene, Phys. Rev. B 89, 195404 (2014).

[34] P. Goswami and S. Chakravarty, Quantum criticality between topological and band insulators in $3+1$ dimensions, Phys. Rev. Lett. 107, 196803 (2011).

[35] P. Hosur, S. A. Parameswaran, and A. Vishwanath, Charge transport in Weyl semimetals, Phys. Rev. Lett. 108, 046602 (2012).

[36] R. E. Throckmorton, J. Hofmann, E. Barnes, and S. Das Sarma, Many-body effects and ultraviolet renormalization in three-dimensional Dirac materials, Phys. Rev. B 92, 115101 (2015).

[37] E.-G. Moon, C. Xu, Y. B. Kim, and L. Balents, NonFermi-liquid and topological states with strong spin-orbit coupling, Phys. Rev. Lett. 111, 206401 (2013).

[38] I. F. Herbut and L. Janssen, Topological Mott insulator in three-dimensional systems with quadratic band touching, Phys. Rev. Lett. 113, 106401 (2014).

[39] B.-J. Yang, E.-G. Moon, H. Isobe, and N. Nagaosa, 
Quantum criticality of topological phase transitions in three-dimensional interacting electronic systems, Nat. Phys. 10, 774 (2014).

[40] H. Isobe, B.-J. Yang, A. Chubukov, J. Schmalian, and N. Nagaosa, Emergent non-Fermi-liquid at the quantum critical point of a topological phase transition in two dimensions, Phys. Rev. Lett. 116, 076803 (2016).

[41] J.-R. Wang, G.-Z. Liu, and C.-J. Zhang, Excitonic pairing and insulating transition in two-dimensional semiDirac semimetals, Phys. Rev. B 95, 075129 (2017).

[42] H.-H. Lai, Correlation effects in double-Weyl semimetals, Phys. Rev. B 91, 235131 (2015).

[43] S.-K. Jian and H. Yao, Correlated double-Weyl semimetals with Coulomb interactions: Possible applications to $\mathrm{HgCr}_{2} \mathrm{Se}_{4}$ and $\mathrm{SrSi}_{2}$, Phys. Rev. B 92, 045121 (2015).

[44] J.-R. Wang, G.-Z. Liu, and C.-J. Zhang, Quantum phase transition and unusual critical behavior in multi-Weyl semimetals, Phys. Rev. B 96, 165142 (2017).

[45] S.-X. Zhang, S.-K. Jian, and H. Yao, Correlated tripleWeyl semimetals with Coulomb interactions, Phys. Rev. B 96, 241111(R) (2017).

[46] J.-R. Wang, G.-Z. Liu, and C.-J. Zhang, Breakdown of Fermi liquid theory in topological multi-Weyl semimetals, Phys. Rev. B 98, 205113 (2018).

[47] Y. Huh, E.-G. Moon, and Y. B. Kim, Long-range Coulomb interaction in nodal-ring semimetals, Phys. Rev. B 93, 035138 (2016).

[48] Y. Wang and R. M. Nandkishore, Interplay between short-range correlated disorder and Coulomb interaction in nodal-line semimetals, Phys. Rev. B 96, 115130 (2017).

[49] J.-R. Wang, G.-Z. Liu, and C.-J. Zhang, Topological quantum critical point in a triple-Weyl semimetal: NonFermi-liquid behavior and instabilities, Phys. Rev. B 99, 195119 (2019).

[50] S. Han, C. Lee, E.-G. Moon, and H. Min, Emergent anisotropic non-Fermi liquid at a topological phase transition in three dimensions, Phys. Rev. Lett. 122, 187601 (2019).

[51] S.-X. Zhang, S.-K. Jian, and H. Yao, Quantum criticality preempted by nematicity, arXiv:1809.10686

[52] S. Han and E.-G. Moon, Long-range Coulomb interaction effects on the topological phase transitions between semimetals and insulators, Phys. Rev. B 97, 241101(R) (2018).

[53] S. Han, G. Y. Cho, and E.-G. Moon, Quantum criticality with infinite anisotropy in topological phase transitions between Dirac and Weyl semimetals, Phys. Rev. B 98, 085149 (2018).

[54] B. Roy, M. P. Kennett, K. Yang, and V. Juričić, From birefringent electrons to a marginal or non-Fermi liquid of relativistic spin-1/2 fermions: An emergent superuniversality, Phys. Rev. Lett. 121, 157602 (2018).

[55] M. D. Uryszek, E. Christou, A. Jaefari, F. Krüger, and B. Uchoa, Quantum criticality of semi-Dirac fermions in $2+1$ dimensions, Phys. Rev. B 100, 155101 (2019).

[56] S. Sur and B. Roy, Unifying interacting nodal semimetals: A new route to strong coupling, Phys. Rev. Lett. 123, 207601 (2019).

[57] I. F. Herbut, Interactions and phase transitions on graphene's honeycomb lattice, Phys. Rev. Lett. 97, 146401 (2006).

[58] I. F. Herbut, V. Juričić, and B. Roy, Theory of interacting electrons on the honeycomb lattice, Phys. Rev. B 79,
085116 (2009).

[59] B. Roy and S. Das Sarma, Quantum phases of interacting electrons in three-dimensional dirty Dirac semimetals, Phys. Rev. B 94, 115137 (2016).

[60] J. Maciejko and R. Nandkishore, Weyl semimetals with short-range interactions, Phys. Rev. B 90, 035126 (2014).

[61] B. Roy, P. Goswami, and V. Juričić, Interacting Weyl fermions: Phases, phase transitions, and global phase diagram, Phys. Rev. B 95, 201102(R) (2017).

[62] B. Roy and M. S. Foster, Quantum multicriticality near the Dirac-semimetal to band-insulator critical point in two dimensions: A controlled ascent from one dimension, Phys. Rev. X 8, 011049 (2018).

[63] J. Wang, Role of four-fermion interaction and impurity in the states of two-dimensional semi-Dirac materials, J. Phys.: Condens. Matter 30, 125401 (2018).

[64] A. L. Szabó, R. Moessner, and B. Roy, Interacting spin$3 / 2$ fermions in a Luttinger (semi)metal: competing phases and their selection in the global phase diagram, arXiv: 1811.12415

[65] S. Sur and R. Nandkishore, Instabilities of Weyl loop semimetals, New J. Phys. 18, 115006 (2016).

[66] B. Roy, Interacting nodal-line semimetal: Proximity effect and spontaneous symmetry breaking, Phys. Rev. B 96, 041113(R) (2017).

[67] M. A. N. Araújo and L. Li, Broken-symmetry phases of interacting nested Weyl and Dirac loops, Phys. Rev. B 98, 155114 (2018).

[68] S. V. Syzranov and B. Skinner, Electron transport in nodal-line semimetals, Phys. Rev. B 96, 161105(R) (2017).

[69] C. Li, C. M. Wang, B. Wan, X. Wan, H.-Z. Lu, and X. C. Xie, Rules for phase shifts of quantum oscillations in topological nodal-line semimetals, Phys. Rev. Lett. 120, 146602 (2018).

[70] W. Chen, H.-Z. Lu, and O. Zilberberg, Weak localization and antilocalization in nodal-line semimetals: Dimensionality and topological effects, Phys. Rev. Lett. 122, 196603 (2019).

[71] Z.-M. Yu, W. Wu, X.-L. Sheng, Y. X. Zhao, and S. A. Yang, Quadratic and cubic nodal lines stabilized by crystalline symmetry, Phys. Rev. B 99, 121106(R) (2019).

[72] L. Li, S. Chesi, C. Yin, and S. Chen, 2 2 -flux loop semimetals, Phys. Rev. B 96, 081116(R) (2017).

[73] R. Shankar, Renormalization-group approach to interacting fermions, Rev. Mod. Phys. 66, 129 (1994).

[74] R. Nandkishore, L. S. Levitov, and A. V. Chubukov, Chiral superconductivity from repulsive interactions in doped graphene, Nat. Phys. 8, 158 (2012).

[75] M. A. Metlitski, D. F. Mross, S. Sachdev, and T. Senthil, Cooper pairing in non-Fermi liquids, Phys. Rev. B 91, 115111 (2015).

[76] O. Vafek and K. Yang, Many-body instability of Coulomb interacting bilayer graphene: Renormalization group approach, Phys. Rev. B 81, 041401(R) (2010).

[77] F. Zhang, H. Min, M. Polini, and A. H. MacDonald, Spontaneous inversion symmetry breaking in graphene bilayers, Phys. Rev. B 81, 041402(R) (2010).

[78] H. Isobe and L. Fu, Supermetal, Phys. Rev. Research 1, 033206 (2019).

[79] P. A. Volkov and S. Moroz, Coulomb-induced instabilities of nodal surfaces, Phys. Rev. B 98, 241107(R) (2018). 